\title{
EchoGéo
}

21 | 2012

Pays émergents

\section{Géographie du changement social en Égypte}

\author{
Delphine Pagès-El Karoui
}

\section{(2) OpenEdition}

Journals

Édition électronique

URL : https://journals.openedition.org/echogeo/13204

DOI : 10.4000/echogeo. 13204

ISSN : 1963-1197

\section{Éditeur}

Pôle de recherche pour l'organisation et la diffusion de l'information géographique (CNRS UMR 8586)

\section{Référence électronique}

Delphine Pagès-El Karoui, « Géographie du changement social en Égypte », EchoGéo [En ligne], 21 | 2012, mis en ligne le 23 novembre 2012, consulté le 10 août 2021. URL : http://

journals.openedition.org/echogeo/13204; DOI : https://doi.org/10.4000/echogeo.13204

Ce document a été généré automatiquement le 10 août 2021.

EchoGéo est mis à disposition selon les termes de la licence Creative Commons Attribution - Pas d'Utilisation Commerciale - Pas de Modification 4.0 International (CC BY-NC-ND) 


\title{
Géographie du changement social en Égypte
}

\author{
Delphine Pagès-El Karoui
}

\section{NOTE DE L'AUTEUR}

La réalisation de cet article a été financée par l'ANR SYSREMO.

1 Le vent du changement qui a soufflé en 2011 sur le monde arabe a été une surprise pour tous. La vision simpliste d'une région figée dans ses permanences, où l'autoritarisme politique garantissait la stabilité, où la société bâillonnée acceptait son apathie avec résignation, cette vision a volé en éclats, cédant la place à une multitude d'images brouillées et parfois contradictoires. Rares sont ceux qui avaient pris conscience de l'ampleur des bouleversements qui ont radicalement transformé, ces dernières décennies, les sociétés et les espaces de la région. Plus grand pays du monde arabe, territoire de haute densité et d'intenses migrations, l'Égypte est emblématique de ces évolutions. En 2010, avec un taux de croissance du PIB de $5 \%$ et des résultats macroéconomiques prometteurs, l'Égypte avait été classée parmi les CIVETS, un acronyme désignant 6 pays (Colombie, Indonésie, Vietnam, Égypte, Turquie et Afrique du Sud ), censés être la deuxième génération de pays émergents après les BRIC (Brésil, Russie, Inde, Chine). Pleinement insérée dans les réseaux de la mondialisation, suite à une libéralisation (infitâh) progressive de son économie depuis les années 1970, l'Égypte dispose de groupes nationaux qui se sont transformés en firmes multinationales, comme le groupe Orascom de la famille Sawires, présent dans les télécommunications, l'hôtellerie et la construction.

2 La libéralisation n'est qu'une facette de ces bouleversements intenses et multiformes qui ont touché la société et la révolution politique du 25 janvier 2011 en est l'une de ces traductions. La révolution démographique qui se manifeste par une baisse de la fécondité est le résultat d'une meilleure éducation de la population, notamment des femmes. Elle s'inscrit dans un bouleversement profond de la société, travaillée par des 
logiques d'individualisation (distanciation et autonomisation de l'individu par rapport au groupe d'appartenance) et de remises en cause des équilibres traditionnels entre les générations et les sexes. La perturbation de ces équilibres peut susciter une désorientation de la société et aboutir à des renversements de l'autorité politique (Courbage, Todd, 2007). Cette lecture s'inscrit dans le "paradigme de la modernité ", qui traverse un grand nombre d'études portant sur le Moyen-Orient (Bozarslan, 2011, p. 23).

3 En Égypte, coexistent plusieurs visions de ces transformations, passées ou à accomplir, de la société. La modernité n'est pas toujours le référent central invoqué : les discours réformistes pensent moins les transformations sociales comme une rupture avec la tradition que comme un moyen de renouer avec un processus de civilisation dont l'Égypte a été historiquement la glorieuse dépositaire à l'époque pharaonique, et qui a fonctionné comme matrice pour les autres civilisations du monde (l'Égypte comme Um al-Dûnia, mère du monde). Au Moyen-Orient, le concept de modernité apparaît plutôt dans les années 1960 dans le cadre "d'une pensée développementaliste euroaméricaine » (Bozarslan, 2011, p. 24), pour décrire l'émergence des classes moyennes, l'essor de la scolarisation et l'industrialisation. Il s'agit de rattraper le retard face à l'Occident, en l'imitant. Cette grille de lecture proche, en un sens, des visions réformistes des intellectuels de la Renaissance arabe (nahda $a^{1}$ de la fin du XIX ${ }^{e}$ siècle, diffère des attentes révolutionnaires, portées par Nasser dont la prise de pouvoir en 1952 signifie la fin de la monarchie et l'instauration de la république. Le discours révolutionnaire impose par le haut les transformations de la société (réforme agraire, nationalisation des entreprises, meilleur accès à l'éducation et aux soins), et propose de rattraper le retard face à l'Occident, via la production d'une modernité endogène (Roussillon, 1992) en combattant l'impérialisme par l'alliance des peuples opprimés et en forgeant le concept de socialisme arabe. Finalement, l'échec de ces deux utopies de la délivrance que sont la réforme et la révolution a laissé le champ libre à l'épanouissement du paradigme islamiste, qui opère un glissement de l'horizon de la nation vers l'Islam, posé comme un nouvel universel, tout en reformulant la question de l'aliénation, résolue par le retour à l'authenticité.

4 Dans cet article, nous préférons mettre en avant des processus de modernisation, afin de s'affranchir des apories de la modernité, quand elle est perçue comme une démarche téléologique inéluctable vers le progrès, lié à un référent unique, l'Occident, et quand elle néglige la prise en compte de l'autoritarisme et des inégalités sociales et économiques des pays en développement. Nous utiliserons aussi un terme proche, le changement social, certes un peu désuet aujourd'hui (Trémoulinas, 2006), mais héritier d'une longue tradition sociologique, de Tocqueville à Durkheim, de Marx à Weber et qui fait référence à toute transformation durable qui affecte la structure ou le fonctionnement d'une société. Décliné au pluriel, ce concept permet, dans le cas égyptien, de dépasser la dichotomie simpliste, entre un universalisme uniformisé forcément occidental et un particularisme universalisant forcément islamiste. Entre les deux, une multitude de chemins, de pratiques et de représentations se dessinent et les processus d'occidentalisation et d'islamisation vont de pair et s'influencent réciproquement.

5 L'autre avantage du concept de changement social consiste à ne pas trancher, dès à présent, le débat récurrent autour de la validité du terme de "révolution" pour qualifier les événements qui ont lieu depuis le 25 janvier 2011 (Adonis, 2011 ; Lavergne, 
2011 ; Pagès-El Karoui, 2012). Peut-on parler de révolution si, malgré la chute de Moubarak, le régime s'est maintenu au pouvoir en dépit de l'élection récente d'un Frère musulman à la présidence de la République, dont le Conseil Suprême des Forces Armées (CSFA), avait pris soin de vider, quelques jours avant sa nomination, de ses principales fonctions? Le limogeage en août 2012 du chef du CSFA par le nouveau président Mohamed Morsi montre que le bras de fer est engagé entre les islamistes et l'armée. Si la révolution n'a pas atteint immédiatement le cœur du système politique, le départ de Moubarak, après celui de Ben Ali, constitue un événement sans précédent, un changement radical pour l'Égypte : le soulèvement transcendant les classes sociales, la libération de la parole politique, le rejet de l'autorité témoignent d'une transformation profonde des mentalités. L'objectif de cet article est ambitieux : il s'agit de tenter d'élaborer une synthèse, qui n'a pas encore été réalisée, sur les relations en amont entre changement social et révolution. Comment rendre compte de la multiplicité et de l'ambivalence des changements sociaux, en les inscrivant dans des processus universels ou régionaux, mais sans sacrifier les spécificités nationales à la force contraignante d'un modèle?

6 La révolution qui constitue la toile de fond de cette étude, ne sera pas évoquée en tant que telle, car elle a déjà fait l'objet d'un précédent article, publié à quatre mains dans Echogéo (Pagès-El Karoui, Vignal, 2011) et que cette contribution vient prolonger ${ }^{2}$. L'impact des événements actuels, créateurs de profonds bouleversements en aval, ne constitueront pas non plus le cœur du propos, car il est bien trop tôt pour disposer d'informations fiables et pour en offrir une lecture cohérente. Pour donner à voir ce changement social, qui est resté invisible pour des nombreux observateurs occidentaux, rendant incompréhensibles et «miraculeux » les soulèvements de 2011, nous reviendrons sur l'individualisation de la société - facilitée par l'urbanisation du territoire -, et ses traductions au niveau de la famille et de la religion. Nous évoquerons ensuite le rôle des mutations économiques (néo-libéralisation, essor de la société de consommation et de loisirs), et quelques unes de leurs traductions spatiales. Enfin, parmi les nombreux acteurs du changement social (État, mouvements sociaux, ONG...), nous interrogerons le rôle d'une catégorie, les migrants, souvent dénoncés dans les médias égyptiens comme les vecteurs d'un changement culturel négatif.

\section{Révolution et changement social}

7 Les processus du changement social en Égypte, comme dans le monde arabe en général, sont imputables pour simplifier, à deux grands types de changement: la transition démographique et la transition économique vers un système libéral. Face à une société largement déstabilisée par ces mutations rapides, l'islamisme politique constitue une réponse satisfaisante pour une majorité d'Égyptiens qui y voient le signe d'une authenticité compatible avec la modernité. La place des femmes dans la société illustre bien toute l'ambivalence des processus en cours.

\section{Urbanisation et individualisation de la société}

\section{Un territoire majoritairement urbanisé}

8 Un élément souvent sous-estimé par les chercheurs en sciences sociales est l'impact de l'urbanisation sur le changement social dans le monde arabe. En Égypte, le territoire, 
longtemps majoritairement rural, s'est profondément urbanisé, en dépit des statistiques officielles qui se refusent à énoncer cette vérité. Dans un contexte de densités extrêmes qui contribuent à brouiller la distinction entre l'urbain et le rural, l'État égyptien maintient une définition extrêmement restrictive de l'urbain, limité aux localités qui ont le statut administratif de chefs-lieux de gouvernorat ou de district. Il tente ainsi de se persuader que l'urbanisation ne progresse pas : de 1960 à 2006, le taux d'urbanisation aurait stagné à $43 \%$ de la population (Pagès-El Karoui, 2008). Cette définition restrictive de l'urbain considère donc comme ruraux tous les anciens villages englobés dans les agglomérations des villes et tous les villages qui ont connu des processus d'urbanisation très marqués. En revanche, si l'on considère la population vivant dans des agglomérations de plus de 10000 habitants, le taux d'urbanisation monte alors à $82 \%$ (Moriconi, 2011), ce qui correspond davantage à la réalité du territoire égyptien. Parallèlement, la structure de la population active s'est elle aussi profondément modifiée, avec le secteur agricole qui ne représente plus qu'environ $25 \%$ de l'emploi total (Denis, 2011a).

9 Avec plus de 2000 habitants par $\mathrm{km}^{2}$, l'Égypte fait figure d'un des pays les plus denses au monde ${ }^{3}$, avec une densité quatre fois plus élevée qu'aux Pays-Bas, par exemple. On retrouve là les éléments d'une analyse durkheimienne du changement social qui reliait l'approfondissement de la division du travail à la densification du territoire. Pour Durkheim, le concept de densité est double : il renvoie à la fois à la densité matérielle augmentation de la densité moyenne de la population-, mais aussi à la densité «morale»-augmentation du nombre de contacts sociaux - (Trémoulinas, 2006). En Égypte, comme ailleurs, l'urbanisation conduit à l'individualisation. Comme l'avait démontré simmel, la ville rend anonyme et affranchit l'individu des communautés traditionnelles (le village, la tribu, la confrérie soufie..). Les villes font figure de laboratoires des nouveaux comportements démographiques: c'est d'abord dans les villes que la fécondité a commencé à baisser.

\section{Transition démographique : le secret des révolutions ?}

10 Avec plus de 80 millions d'habitants et une population qui augmente de plus d'un million de personnes chaque année, l'Égypte constitue une exception démographique. Alors que la Tunisie, le Liban ou l'Iran ont atteint un taux de fécondité inférieur au renouvellement des générations (2,1 enfants par femme), la transition démographique en Égypte reste inachevée, avec 3 enfants par femme en 2010 (contre 5 en 1980). La chute de la fécondité n'a pas été linéaire, mais a suivi les grandes tendances économiques régionales: elle a commencé à baisser dans les années 1960, pour remonter ensuite dans les années 1970, soutenue par la croissance économique, due à une rente pétrolière indirecte. Dans les années 1990, les politiques d'ajustement structurel ont coïncidé avec une nouvelle chute de la fécondité, avant de stagner aujourd'hui de nouveau. La chute de la fécondité a donc d'abord commencé dans les grandes villes puis s'est propagée au monde rural : en 2008, l'écart de fécondité est devenu faible entre milieux urbain et rural (2,7 contre 3,2 enfants par femme). En revanche, le « retard » du rural de la Haute-Égypte est plus marqué avec 3,6 enfants par femmes. Ces chiffres permettent de rappeler que si le clivage urbain/rural se réduit en Égypte, avec la convergence des comportements démographiques, le décrochage du Saî̀ (Haute-Égypte) demeure préoccupant, cette région cumulant les records nationaux de pauvreté et de sous-développement. 
Tableau 1 - Indice de fécondité selon les grandes régions d'Égypte

\begin{tabular}{|l|l|}
\hline Régions & Indice synthétique de fécondité (nombre d'enfants par femme) \\
\hline Gouvernorats urbains & 2,6 \\
\hline Delta urbain & 2,6 \\
\hline Delta rural & 3 \\
\hline Vallée urbain & 3 \\
\hline Vallée rural & 3,6 \\
\hline Gouvernorats de la frontière & 3,3 \\
\hline Égypte urbain & 2,7 \\
\hline Égypte rural & 3,2 \\
\hline Égypte & 3 \\
\hline
\end{tabular}

Source : Egyptian Demographic and Health Survey (EDHS), 2008 (Ambrosetti, 2011)

11 Cette baisse de la fécondité est à mettre en relation avec la hausse du niveau d'éducation de la population, au moins pour ses classes d'âge les plus jeunes (le taux d'alphabétisation en 2006 est de $71 \%$ ) et plus particulièrement, l'alphabétisation des femmes, même s'il demeure un analphabétisme féminin à éradiquer, en particulier dans les zones rurales de la Haute-Égypte. Aujourd'hui, 56 \% des étudiants diplômés de l'université sont des femmes. Parce qu'elles font davantage d'études et qu'elles se marient plus tard, les femmes ont moins d'enfants.

Sous Nasser, fut lancée une vaste politique d'éducation massive afin d'offrir une éducation gratuite pour tous, une large partie du budget étant consacrée à l'éducation. Mais, dès le milieu des années 1970 , l'école peinait à répondre à la massification de l'enseignement imposée par la croissance démographique. Depuis, la situation a empiré. Faute de locaux disponibles, les écoles publiques sont surchargées et se développe le système de la double (voire la triple) période, c'est-à-dire que les mêmes locaux sont occupés par des enfants différents sur différentes tranches horaires. Parallèlement, se mettent en place les cours particuliers : pour augmenter leur salaire dérisoire, les professeurs des écoles publiques instituent des cours du soir, quasiment «obligatoires » pour pouvoir obtenir de bonnes notes dans le cours officiel. Le prix de ces cours « particuliers » collectifs (ils peuvent rassembler la quasi-totalité de la classe) grèvent considérablement le budget des ménages, quand ils parviennent à les financer. On assiste donc à une sorte de privatisation informelle de l'éducation publique, tandis que les classes aisées placent massivement leurs enfants dans des écoles privées qui misent souvent sur la pratique des langues étrangères.

13 L'Égypte compte aujourd'hui une vingtaine d'universités publiques (depuis les années 1970, de nombreuses universités publiques ont été ouvertes en province, notamment dans le Delta et le canal de Suez) et une trentaine d'universités privées qui se sont 
multipliées depuis le milieu des années 1990, s'installant en priorité dans les villes nouvelles autour du Caire. Nasser avait offert à tout diplômé de l'université un emploi dans la fonction publique dont la main-d'œuvre est rapidement devenue pléthorique. Cette promesse a dû être abandonnée, dès les années 1980, suscitant régulièrement la colère des jeunes diplômés. L'économie égyptienne, même avec des taux de croissance du PIB supérieurs à $5 \%$ par an, ne parvient pas à trouver du travail pour les 2 millions de nouveaux arrivants qui se déversent chaque année sur le marché du travail. Le chômage des jeunes diplômés s'est donc considérablement aggravé en Égypte, et même pour ceux qui parviennent à trouver un emploi, les salaires sont généralement très bas, ce qui pousse une grande partie de ces jeunes à vouloir s'expatrier pour améliorer leurs conditions de vie.

14 De nombreux chercheurs expliquent ce fort taux de chômage, notamment par la mauvaise qualité de l'enseignement et rappellent qu'en termes d'impact de l'éducation sur le changement social, «il convient de distinguer entre accès à l'éducation, scolarisation et achèvement de la scolarité d'un côté, et qualité, contenu et impact socioculturel et politique de la scolarisation de l'autre» (Herera, 2011, p. 692). Les organisations internationales (UNESCO, UNICEF, PNUD, la Banque mondiale et les agences de développement américaine (USAID), européenne, canadienne et japonaise) considèrent l'éducation de la population égyptienne comme un enjeu prioritaire. Leur intervention, notamment sur le contenu des programmes a suscité de larges débats lorsque l'USAID a incité à remplacer les cours de religion obligatoires et séparés pour les musulmans et les chrétiens par un cours sur les valeurs morales, les critiques dénonçant unanimement une volonté américaine de participer à la sécularisation de la jeunesse égyptienne (id.).

\section{Les transformations de la famille}

La transition démographique modifie les fondements de la famille patriarcale, fondée sur la famille élargie et la double domination des hommes sur les femmes, et des plus âgés sur les plus jeunes. La famille nucléaire devient la norme qui se diffuse aussi en milieu rural (Louhichi, 1997). Cette modernisation à l'œuvre ne doit pas faire croire à une convergence à l'identique avec les sociétés européennes: les formes de la modernisation ne sont pas identiques d'une société à l'autre. Le mariage reste l'institution centrale de la société égyptienne, avec son corrélat, l'absence théorique de relations sexuelles avant l'union officielle. Ainsi, le taux définitif de célibat à 45-49 ans représente moins de $2 \%$ de la tranche d'âge (Fargues, 2011). Avec le recul de l'âge du mariage, le célibat chez les trentenaires se développe (Ambrosetti, 2011). Une autre tendance va à l'encontre des processus observés en Europe : le divorce, par exemple, a beaucoup reculé. Dans les années 1930, il concernait un mariage sur deux contre seulement $13 \%$ en 2008. Autrefois, on divorçait beaucoup pour se remarier ensuite, tandis qu'aujourd'hui, la norme sociale impose une plus grande stabilité conjugale. Dernier exemple: si la décohabitation des jeunes ménages est un fait de plus en plus avéré (le couple n'habite plus forcément comme le voulait la tradition chez les parents du mari, dans le cadre de la famille élargie), les jeunes célibataires, garçons ou filles, habitent toujours chez leurs parents. Dans un pays profondément pieux et conservateur, la famille reste la cellule de base de la société et sert de modèle fondateur pour l'imaginaire national (Baron, 2007). 


\section{Le retour du religieux}

\section{Modernisation et islamisation} phénomène a été théorisé pour l'Europe par Marcel Gauchet (1985) avec le concept de "sortie de religion" qui signifie moins le déclin de la croyance religieuse comme conviction personnelle que le fait que l'individu cesse de considérer la religion comme la norme englobante de son action. Or, depuis les années 1970, la tendance est plutôt inverse en Égypte : on assiste à un retour du religieux dans l'espace public qui avait été, non pas occulté, mais mis au second plan pendant les années nassériennes du socialisme arabe. Cette réislamisation ${ }^{4}$ se manifeste par la généralisation du port du voile pour les femmes, le port de la barbe et de la "zébiba " pour les hommes, la multiplication des chaines de télévision religieuses et des mosquées dont les puissants haut-parleurs marquent une forte emprise sonore sur l'espace public, une plus grande visibilité de la prière, notamment sur les lieux de travail, un respect plus sévère du Ramadan...

Cette réislamisation a été portée dans un premier temps par les Frères musulmans, dont la création remonte à la fin des années 1920, et dont l'idéologie apparaît clairement comme un mouvement paradoxal, inventeur d'une modernité authentiquement égyptienne en réaction à l'occidentalisation et l'individualisation de la société, portées par les changements démographiques. Sous le régime de Moubarak, les Frères musulmans constituaient la première force d'opposition structurée. Ils ont su remporter l'adhésion d'une partie de la population égyptienne en se substituant, dans les quartiers informels, à l'absence de l'État, en prenant en charge via des associations locales de bienfaisance des services «publics » comme : le registre de l'état-civil, des dispensaires, des cours particuliers, des séances d'alphabétisation, la prise en charge des veuves et orphelins.

Toutefois, la réislamisation n'a pas été le seul fait des militants islamistes, elle a aussi été largement orchestrée par les gouvernements. Dans les années 1970, Sadate s'en est servi afin d'affaiblir son opposition de gauche et faire passer le renversement des alliances (URSS/États-Unis) et du système de valeurs que sa politique d'ouverture imposait (arabisme/islamisme). En 1971, la charia est devenue, d'après la Constitution, l'une des principales sources du droit. En 1972, les conseillers russes ont été renvoyés et en 1979, la paix avec Israël a été signée, en échange d'une importante aide financière des États-Unis, aide qui perdure jusqu'à maintenant. Moubarak n'a rien changé au système, il l'a juste prolongé en accentuant les réformes libérales dans un pays qui avait maintenu une partie du système économique hérité de Nasser.

La population égyptienne reste dominée par un conservatisme social, dont la norme est un islam dépolitisé, ou une "copticité » dépolitisée car les coptes sont tout autant concernés par le retour du religieux. L'objectif de ce conservatisme est de « renforcer les repères de solidarités ", de redéfinir "les rapports sociaux à partir des liens de dépendance des individus vis-à-vis d'une autorité (patriarcale, religieuse ou de classe)", de créer "l'illusion d'une possible dépolitisation de ces rapports pour surmonter la crise économique et sociale [...] par le simple surinvestissement disciplinaire et moral»(Bozarslan, 2011, p. 76). Ainsi, le retour à la religion de la société égyptienne ne constitue pas un retour à la tradition. Il s'agit bien pour les Égyptiens d'inventer des voies d'accès à la modernité qui leur soient propres et qui ne 
sont pas forcément incompatibles avec une certaine «américanisation» des modes de vie, même si, paradoxalement, l'image de l'Amérique est très dégradée en Égypte en raison de sa politique étrangère (soutien d'Israël, occupation de l'Irak). Cette influence se retrouve surtout chez les classes les plus aisées, où l'on rencontre de jeunes femmes voilées d'un foulard luxueux et chatoyant, rêvant d'Amérique et parlant anglais à leurs enfants.

Progressivement, cette réislamisation initiée par les Frères Musulmans puis soutenue par le pouvoir en place a échappé à ces deux acteurs pour se constituer en « un marché religieux dont plus personne n'a le monopole. [...] Bref, aussi paradoxal que cela puisse paraître, la réislamisation a entraîné une banalisation et une dépolitisation du marqueur religieux : quand tout est religieux, plus rien n'est religieux. » (Roy, 2011). Dans ce contexte, a émergé le concept de post-islamisme qui désigne d'une part le constat d'échec de l'islam politique et d'autre part, l'autonomisation des dynamiques sociales vis-à-vis de l'État existant, même si certains discours peuvent véhiculer une rhétorique islamique. Il invite à penser «l'apparition d'un espace de laïcité dans les sociétés musulmanes, non pas du fait du recul de la croyance ou de la pratique, mais parce que le champ religieux tend à se dissocier du champ politique » (Roy, 1999a). Ce terme inventé par Asef Bayat pour l'Iran puis élargi à l'ensemble des sociétés musulmanes $(1996,2010)$, a été repris par Olivier Roy (1999b), puis radicalisé par Gilles Kepel (2000) en une fin de l'islamisme. Son emploi a suscité bien des controverses parmi les politologues (Burgat, 2001). Ce n'est pas le lieu ici de rentrer dans le débat qui a surtout porté sur la réalité du déclin de l'islam politique, et sur le fait que les dynamiques de réislamisation et de modernisation étaient d'emblée compatibles, même dans la première phase de l'islam politique. L'important pour nous est leur accord sur le fait que «l'islamisation n'implique pas nécessairement la mise en place d'un autre modèle de société, mais plutôt la réappropriation du changement » (Roy, 1999b, p. 19).

\section{La recomposition du champ religieux}

Dans ce contexte d'intense recomposition du champ religieux, l'islam sunnite égyptien est loin d'être monolithique. Il existe un renouveau confrérique, certaines pratiques populaires comme la visite au tombeau des saints, les pèlerinages, se perpétuent en dépit de nombreuses attaques dont elles font l'objet (Pagès-El Karoui, 2005). Dans un autre registre, Patrick Haenni (2005) a bien décrit l'avènement en Égypte, tout comme en Turquie ou en Indonésie, de cet « islam de marché » qui a conquis une partie des classes moyennes et de la bourgeoisie. Il s'agit de proposer une nouvelle vision de l'islam, conservateur mais ouvert au monde, qui permet de concilier la réalisation de soi et la quête du salut avec le culte de la performance économique, ou dit autrement d'incarner la religion dans le désir d'individualisme, l'essor de la société de consommation et l'idéologie néo-libérale. Son icône la plus célèbre, Amr Khaled, jeune prédicateur "branché », star des talk-shows religieux, inspiré des télé-évangélistes américains, a ringardisé tant les clercs officiels d'al-Azhar que les militants islamistes (dont il est lui-même issu). Son ambition est de "réarticuler la nahda autour des valeurs de l'entreprise " : ainsi, il ne voit pas en l'Occident l'ennemi de l'islam mais s'inspire au contraire de ses techniques managériales pour rendre le monde arabe de nouveau compétitif et lancer un réseau de projets de développement qui ont rencontré un grand succès. Le génie de Amr Khaled a été «de se positionner au carrefour de trois tendances: l'islamisation des sociétés, la privatisation des États et la globalisation du 
monde " (Haenni, 2006), tout en articulant désir d'individualisme et appartenance nationale. Amr Khaled qui a, très tôt, su jouer des nouvelles technologies de communication - son site personnel est l'un des plus fréquentés au monde - a d'ailleurs immédiatement soutenu les soulèvements en Égypte, en se rendant plusieurs fois place Tahrir.

Face à ce libéralisme post-islamiste, se renforcent les courants néo-fondamentalistes, auxquels se rattachent les salafistes. Il ne s'agit plus là de penser l'islam dans la modernité mais de définir l'islamité, par un retour aux origines - les premiers temps du prophète -, comme un code d'interdits et d'obligations à respecter scrupuleusement, dans l'imitation des pieux ancêtres (salaf-s). Implanté en Égypte dans les années 1970, le salafisme s'est est fortement inspiré du wahhabisme, vision particulièrement rigoriste de l'islam, pratiquée en Arabie Saoudite. Le gouvernement de Moubarak n'avait pas hésité à instrumentaliser les salafistes pour affaiblir les Frères musulmans, avec l'aide de l'Arabie Saoudite qui soutient aussi financièrement sa diffusion hors de ses frontières. Les révolutions arabes ont eu un impact considérable sur la transformation de ce courant, centré au départ sur une individualisation de la religiosité et marqué par un rejet de l'État et de la démocratie. Contre toute attente, les salafistes ont créé des partis et se sont lancés dans la course aux législatives en janvier 2012.

23 Alors que les Frères musulmans n'ont pas initié les soulèvements - les salafistes les avaient même ouvertement condamnés - et que les révoltes ne se sont pas inscrites dans un répertoire de revendications islamiques, les islamistes sont actuellement les grands gagnants des révolutions arabes. En Égypte, ils ont obtenu $70 \%$ des voix aux élections législatives, en additionnant les partis issus des Frères musulmans (45\%) et des salafistes ( $25 \%$ ), au détriment des partis libéraux et révolutionnaires. La surprise est venue de l'importance acquise par les salafistes qui ont réussi à remporter un quart des suffrages. Comment expliquer un tel succès que l'on n'avait pas vu venir? Les interprétations proposées sont nombreuses. Tariq Ramadan ${ }^{6} \mathrm{y}$ voit la main cachée de l'Arabie Saoudite qui, grâce à ses pétrodollars, se fait le champion de la contrerévolution. Les milieux de gauche y décèlent l'action des services secrets égyptiens qui cherchent à affaiblir les Frères musulmans. Selon Stéphane Lacroix ${ }^{7}$, l'aspect inexpérimenté - jusqu'à très récemment ce mouvement quiétiste jugeait l'islam incompatible avec la démocratie et rejetait les élections démocratiques - a paradoxalement été attractif pour les électeurs, en quête de candidats neufs, face aux Frères musulmans qui faisaient figure d'opposants attitrés sous le régime de Moubarak.

Les Frères musulmans sont eux-mêmes divisés entre la base des jeunes militants très actifs sur la place Tahrir et le sommet qui a tenté de négocier avec le pouvoir afin d'obtenir la reconnaissance officielle dont ils étaient privés jusqu'à présent. Un certain nombre de ces jeunes ont d'ailleurs créé des partis autonomes. Le parti Liberté et Justice, issu de la confrérie, est donc tiraillé entre une alliance avec les salafistes qui professent un islam bien plus radical et fanatique (en particulier, au sujet des femmes ou des coptes) $)^{8}$ ou bien avec les libéraux. Avec l'élection d'un Frère musulman à la présidence de la République, engagé dans un bras de fer constant avec l'armée, il semble que la deuxième option soit la plus réaliste. 


\section{La fracture communautaire}

bahaïsme (Legeay, 2007) - et des conversions recule fortement, contrairement à ce qui se passe en Europe (Trémoulinas, 2006). La fracture communautaire se renforce avec la montée des discriminations et la multiplication des actes de violence envers les coptes (cf. illustration 3). Ces derniers vivent de plus en plus repliés sur leurs propres structures communautaires, centrées sur l'église, et se perçoivent comme des citoyens de seconde zone, ce qui a poussé un grand nombre d'entre eux à l'exil, vers les ÉtatsUnis, le Canada ou l'Australie. Pendant la révolution, de nombreux jeunes coptes n'ont pas respecté les exhortations de leur pape Shenouda, inféodé au régime de Moubarak, à ne pas descendre dans la rue. La place Tahrir a célébré l'unité retrouvée des Égyptiens avec le symbole des coptes et musulmans priant tour à tour sur la place Tahrir, se protégeant les uns les autres. Mais, cette union est menacée d'une part par le gouvernement qui l'instrumentalise, en dépit d'un discours sur l'union nationale, pour raviver les clivages (en octobre 2011, l'armée a réprimé brutalement une manifestation copte, tuant 25 personnes) et les salafistes qui ont pris plusieurs fois pour cibles des églises ou des chrétiens.

\section{L'ambivalent statut de la femme}

Le statut des femmes a beaucoup évolué lors des quatre dernières décennies : elles ne sont plus confinées à l'espace domestique par un système patriarcal, fortement érodé par la transition démographique. Ainsi, le fossé entre les hommes et les femmes se réduit : les avancées législatives en termes de divorce, de nationalité - une nouvelle loi permet à des Égyptiennes mariées à des étrangers de transmettre la nationalité à leurs enfants -, de représentation politique - un quota de femmes au Parlement avait été instauré en 2010. Si leur taux d'activité, une fois mariées, reste très bas par rapport à la moyenne mondiale, les difficultés économiques poussent de plus en plus de femmes à travailler (quand elles arrivent à obtenir un emploi) pour assurer un deuxième revenu au ménage. Cependant, dans tous les classements internationaux sur la participation des femmes à la vie politique et économique, l'Égypte demeure très mal placée (Herera, 2011, 691). Des cinéastes ont alerté l'opinion publique sur les violences faites aux femmes (violence conjugale dans Femmes du Caire de Yousry Nasrallah, harcèlement sexuel dans Les femmes du bus 678 de Mohamed Diab).

Si les femmes ont participé activement aux manifestations qui ont détrôné Moubarak, leur place au sein de l'espace public a été fortement menacée dans les mois qui ont suivi (tests de virginité conduits par l'armée sur les manifestantes, agressions sexuelles collectives sur la place Tahrir...). Via leurs salons islamiques, certaines femmes éduquées et pieuses, sont elles-mêmes les fers de lance d'une «salafisation " par le bas de la bourgeoisie cairote et "refusent catégoriquement d'investir l'espace public qu'elles délèguent définitivement aux hommes, conformément à leur représentation segmentaire et sexuée de l'espace » (Nehaoua, 2010, 64).

L'exemple du foulard islamique (higâb) illustre bien la banalisation des marqueurs de l'islamisation. Initialement porté par des jeunes étudiantes sur les campus, le higâb était vécu comme un signe d'engagement politique mais aussi de modernité, en double rupture, avec le voile traditionnel de leurs mères et avec un mode vestimentaire sécularisé. Avec la généralisation de son port, le voile perd sa fonction de marqueur 
identitaire, se dépolitise, se diversifie et se plie aux canons de la mode internationale. Apparaît le voile à l'« espagnole ", léger, aux couleurs chatoyantes assorties à une tenue qui peut être très moulante. Pour celles qui entendent se distinguer, le recours au voile intégral (niqâb) s'impose comme un nouveau marqueur social d'une islamisation plus radicale. Cette quête de formes de religiosité plus "pures" ne se comprend qu'en réaction à une "islamisation qui résiste mal à la tentation du monde " (Haenni, Tammam, 2011, 898).

\section{Mondialisation et changement social}

Nous aimerions revenir ici sur la grande perméabilité du changement social en Égypte aux influences extérieures et à la circulation et la réinterprétation des normes et modèles culturels globalisés 9 . Sans que l'on puisse conclure à une homogénéisation culturelle mondiale, les changements que l'on va décrire ici, ne sont pas propres à l'Égypte, et sont communs à de très nombreux pays émergents, moyen de souligner une fois de plus, l'inanité de la thèse de « l'exceptionnalisme moyen-oriental ».

\section{Libéralisation et essor de la société de consommation}

L'une des principales traductions de la libéralisation a consisté en l'essor d'une véritable société de consommation, en contraste avec la période nassérienne socialiste, durant laquelle des biens fonctionnels, de qualité médiocre et sans souci du design, étaient produits par les entreprises publiques. Au milieu des années 1990, les grandes enseignes internationales du prêt-à-porter (Naf-Nat, Benetton, Stefanel) ou de la restauration rapide (Mc Donalds, KFC, Pizza Hut) ont multiplié les ouvertures de boutiques, privilégiant d'abord les grandes métropoles, Le Caire et Alexandrie, et les espaces touristiques, puis s'implantant ensuite progressivement dans les villes secondaires, notamment celles dotées d'universités pour cibler un public jeune (Pagès, Vignal, 1998). Alors que le marché de l'alimentation était dominé par les petites épiceries de quartier, les années 2000 ont été celles de la grande distribution. Avec cinq hypermarchés et cinq supermarchés (Carrefour Market), Carrefour est présent dans le Grand Caire, à Alexandrie et à Sharm al-Shaykh dans le Sinaï. La société appartient à une joint-venture entre Carrefour SA et le grand groupe de Dubaï, Majid al-Futtaim, qui a introduit le concept d'hypermarché au Moyen-Orient en 1995 et est depuis leader sur le marché. 


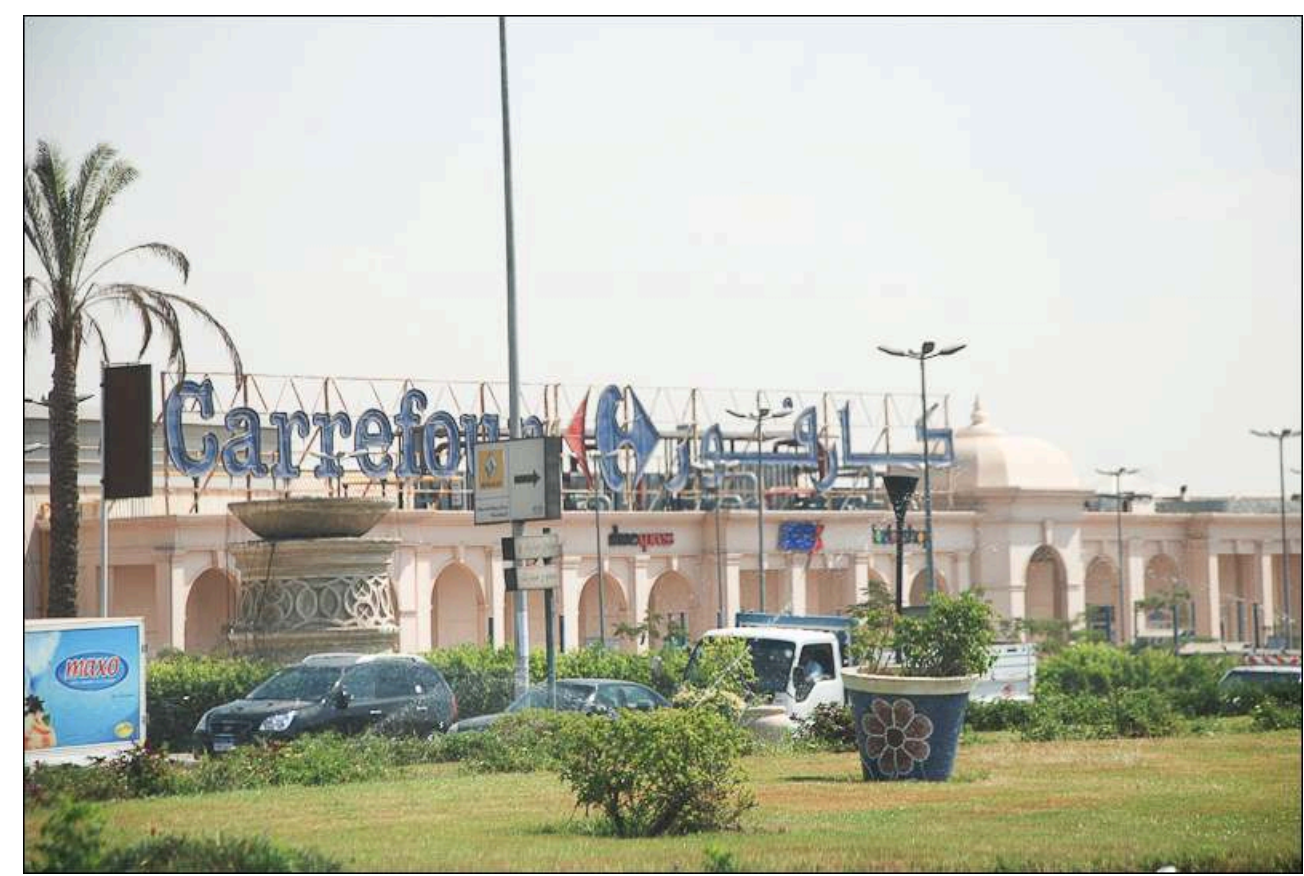

Auteur : D. Pagès-El Karoui, septembre 2011 (ANR SYSREMO).

31 Au regard des statistiques mondiales, l'Égypte apparait comme un pays modérément inégalitaire, même si les consciences de classe sont très marquées. Actuellement, environ $40 \%$ d'Égyptiens vivent en-dessous du seuil de pauvreté. La pauvreté connait une évolution en dents de scie. Pendant les années 1970, en liaison avec le boom pétrolier, la pauvreté avait reculé et les inégalités s'étaient réduites. Entre 1982 et 1995 , ces deux variables ont ensuite augmenté pour rebaisser durant la période 1995-2000 et augmenter de nouveau dans la décennie suivante (Clément, 2011). Alors que le clivage urbain/rural n'est plus si pertinent à l'échelle nationale, le clivage régional lui s'affirme, avec le décrochage de la région du Sa'îd (vallée du Nil), que nous avons déjà souligné.

La vie quotidienne est devenue un calvaire pour les classes moyennes et populaires. Non seulement étudier et trouver un emploi sont devenus compliqués, mais le mariage, institution centrale de la société égyptienne, nécessite l'achat d'un logement équipé par le mari, ce qui explique que de nombreux jeunes émigrent pour pouvoir amasser un pécule et acheter un appartement pour loger leur famille. Compte tenu de l'exigüité de l'œkoumène, les prix du foncier sont très élevés en Égypte. Les jeunes ont alors tendance à acheter ou louer des logements dans les périphéries des villes, bien souvent dans les quartiers informels où ils trouvent un habitat à la mesure de leurs moyens. La liste des difficultés quotidiennes ne serait pas complète si on oubliait la question de la santé (où se développe comme pour l'éducation un système à deux vitesses), de la mobilité (malgré le fort subventionnement des prix de l'essence, ceux-ci augmentent, grevant le prix des transports en commun dont dépendent la majorité de la population) et de l'alimentation qui, avec la forte inflation des produits alimentaires, demeure l'un des sujets de préoccupations essentielles des Égyptiens, pouvant les conduire à la révolte, comme en atteste les «émeutes du pain » en 2008. Bref, la libéralisation a été 
vécue comme un renforcement des inégalités et une paupérisation de la classe moyenne.

Romanciers et cinéastes égyptiens souvent inspirés par le marxisme se sont souvent faits les pourfendeurs de cette société née de l'infitâh, perçue comme le moment de l'effondrement des valeurs traditionnelles - la famille, le savoir-, gangrénées par l'argent facile. Dans son roman Les années de Zeth, qu'il situe dans les années 1980, Sonallah Ibrahim en fait son thème principal. «La marche de la destruction et de la construction " veut que, dans l'immeuble de Zeth, toute personne qui s'enrichit, soit grâce à l'expatriation, soit dans le cadre de la libéralisation (en lien avec l'étranger mais sans quitter le pays), s'engage frénétiquement dans la rénovation de son appartement, en achetant des produits électroménagers de marque étrangère et en ajustant la décoration aux nouveaux standards de la mode. Un des leitmotivs du roman est la frustration de Zeth qui ne peut s'offrir ces améliorations. Vingt ans plus tard, les frustrations des Égyptiens n'ont fait que croître tant pour un grand nombre, l'accès à ces biens de consommation reste hors de portée de leurs bourses. Leurs désillusions sont d'autant plus amères, qu'ils ont acquis une grande familiarité avec ces produits dont les images déferlent sur leurs écrans de télévision ou d'ordinateur.

\section{Une société connectée au monde}

La société égyptienne est bien connectée au monde, via les nouveaux médias ou les incessantes circulations de ses expatriés (cf. infra). L'usage du téléphone portable s'est généralisé et devance dorénavant très largement le téléphone fixe: 76,5 millions d'abonnements contre seulement 9,3 millions en $2011^{10}$. Introduit en 1993, Internet s'est ensuite développé très rapidement, sous la houlette du gouvernement qui a encouragé la démocratisation de son accès via plusieurs initiatives (Guaaybess, 2011). Parallèlement, les cybercafés se sont diffusés sur l'ensemble du territoire égyptien, et Internet parvient jusqu'aux villages, grâce à des connections piratées et partagées qui amoindrissent le coût de la connexion. Aujourd'hui, on estime à 24 millions le nombre d'usagers d'Internet tandis que Facebook enregistre 9,4 millions d'utilisateurs (ce chiffre a doublé pendant l'année 2011) ${ }^{11}$. Le nombre de blogs a explosé après 2003, quand il a été facile de les rédiger en arabe. Les bloggeurs ont été très actifs dans la dénonciation des exactions du régime. Si l'usage d'Internet renvoie à des activités politiques ou de loisirs (musique, jeux...), la principale activité demeure de nature religieuse, pour les jeunes comme pour les moins jeunes: on y écoute les derniers sermons de son prédicateur préféré, et surtout on y cherche des conseils (fatwa), en réponse à des interrogations pratiques sur comment mener une vie moderne, tout en se conformant aux préceptes de l'islam. 


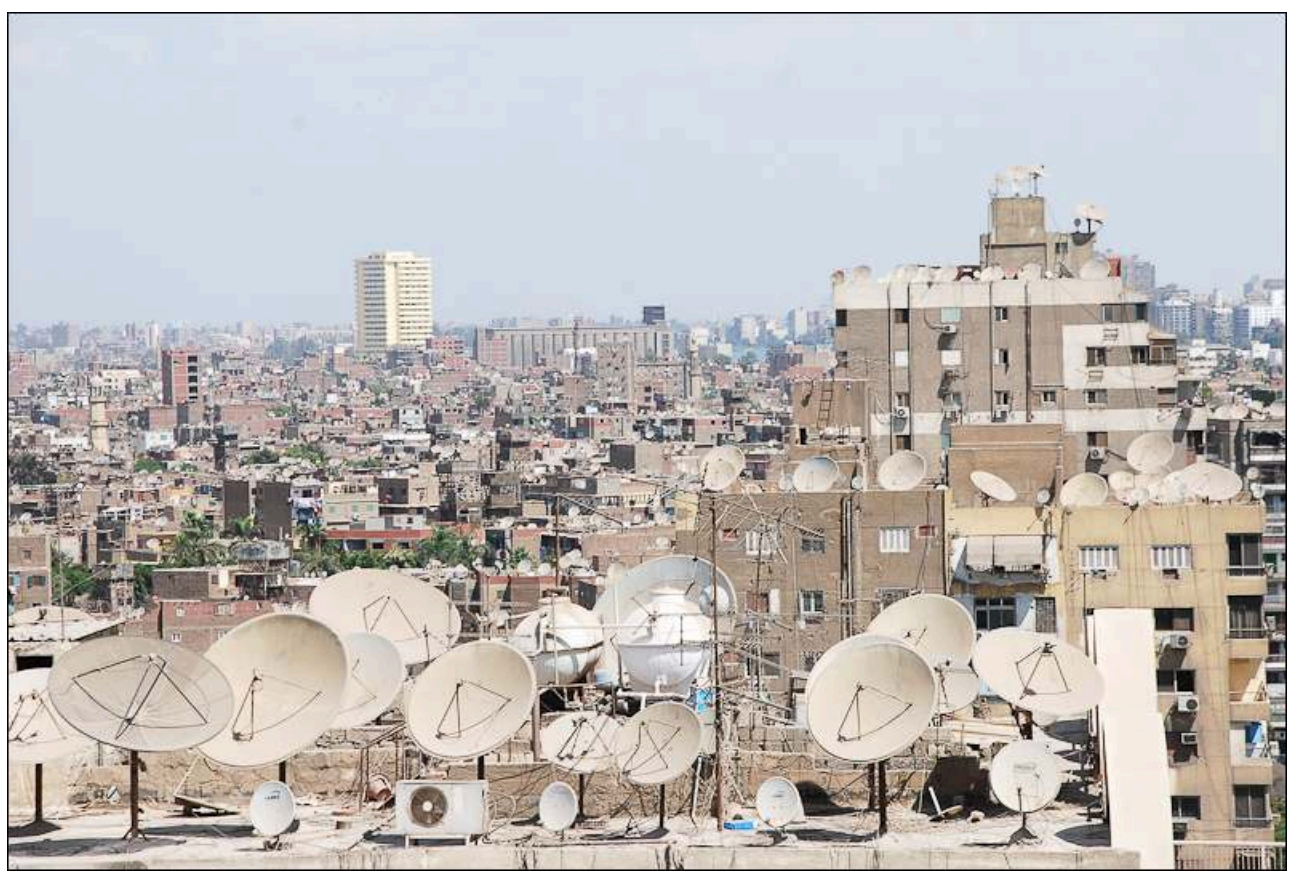

Auteur : D. Pagès-El Karoui, septembre 2011 (ANR SYSREMO).

Les chaines satellitaires sont aussi apparues au début des années 1990 : l'impact de la guerre du Golfe a été très net sur la multiplication des antennes paraboliques. C'est d'abord la chaîne américaine CNN qui a capté l'audience, en offrant un type de programme inédit au public égyptien qui s'affranchissait alors du monopole étatique sur l'information. Depuis, elle a été supplantée par la chaine qatarie Al-Jazira, la plus regardée en Égypte et au Moyen-Orient. Pendant la révolution égyptienne, la chaîne a été la première à émettre des séquences filmées par des portables qui circulaient déjà sur le web, sa couverture ayant été clairement pro-manifestants.

L'impact social de ces nouveaux médias est crucial, car ils ont radicalement transformé les rapports usagers/médias et surtout les rapports État/société civile, en créant l'avènement d'un espace public à la fois égyptien et arabe (Gonzalez-Quijano, Guaaybess, 2009). La contribution de ces nouveaux médias au changement social est fondamentale : ils permettent un accès individuel à l'information, une autonomisation de la connaissance et de la formation du jugement, en défiant les formes d'autorité traditionnelle, familiale ou étatique (Khamis, 2010). S'il n'est pas le lieu de développer ici le débat sur leur rôle pendant la révolution (Ayari, 2011; Zhuo, Wellman,Yu, 2011), rappelons simplement qu'ils ont permis la circulation d'informations en temps réel que le pouvoir n'était plus en mesure de censurer. Ils ont créé une véritable capacité de mobilisation politique en réseaux, très éloignée du fonctionnement traditionnel des partis, autour d'acteurs aussi divers qu'un prédicateur musulman branché (Amr Khaled), d'un opposant politique (Mohamed El Baradeï), ou d'un jeune bloggeur victime de la répression brutale du régime (Khaled Saïd ${ }^{12}$ ). La mobilisation sur Internet a contribué à créer des centralités virtuelles qui, pour réussir à s'imposer dans un rapport de force qui menace réellement le pouvoir, ont dû s'incarner dans l'espace physique de la ville (Pagès-El Karoui, 2012). 


\section{Paysages de la mondialisation}

$37 \mathrm{Au}$ système économique de la libéralisation, correspondent de nouvelles formes urbaines, caractéristiques des pays émergents : verticalisation du bâti, requalification des espaces centraux (aménagement des rives du Nil, rénovation du centre-ville, de la gare centrale Ramsis), processus de patrimonialisation des centres anciens, émergence en périphérie de nouvelles centralités urbaines (villes nouvelles et gated comunities), essor de la spéculation dans les quartiers irréguliers (Denis, 2011b) et construction de nouvelles infrastructures de transports (ringroad du Caire, aéroports construits en BOT $^{13}$ ), multiplication des zones franches, apparition de nouveaux lieux (cybercafés, fast-foods, hypermarchés...). Comme partout ailleurs, les dirigeants ont cherché à adapter l'espace urbain aux nouvelles normes métropolitaines: en 2008, Gamal Moubarak, le fils d'Hosni Moubarak, avait piloté le projet « Grand Caire 2050 » qui visait à inscrire la capitale au rang des « villes mondiales ». Pour bâtir ces nouveaux quartiers vitrines de la mondialisation, plusieurs sites avaient été identifiés, essentiellement des quartiers informels (Imbaba, face à l'île très chic de Zamalek, Nezlet al-Semman à proximité des pyramides de Giza), ce qui impliquait de nombreuses opérations de destruction et de relogement. Ce projet qui a suscité la colère des habitants et a déclenché un débat dans la presse nationale, soulevé par des experts en urbanisme, a finalement été suspendu quelques mois après la chute de Moubarak (Barthel, 2011). Sans prétendre être exhaustif, nous présenterons ici trois types de ces paysages urbains nés de la mondialisation.

\section{Villes nouvelles, malls et gated communities}

Les villes nouvelles égyptiennes répondaient au départ à la volonté d'apporter une réponse à la crise du logement, de déconcentrer la capitale et de détourner la croissance urbaine, souvent informelle des terres agricoles si précieuses pour un territoire à $95 \%$ désertique. Comme souvent en Égypte, ces formes urbaines s'inscrivaient dans un dessein plus large de réforme sociale et de "renaissance nationale"; il s'agissait de proposer un nouveau modèle de citadinité, tout en modernisant la société (Florin, 2005). Si dans les années 1990, leur succès se manifestait davantage par la réussite de quelques zones industrielles autour du Caire (Six-Octobre, Dix-de-Ramadan), les résultats en termes résidentiels étaient moins probants : elles ne comptaient que 300000 habitants sur les 6 millions prévus. Finalement, c'est grâce à une transformation radicale du projet initial dans les années 2000 , que les villes nouvelles ont réussi à devenir réellement attractives ${ }^{14}$. Les promoteurs immobiliers ont réussi à attirer les classes supérieures, en leur vendant un nouveau mode de vie, dans un univers urbain, calme, vert, équipé de tous les services nécessaires, bref, tout ce qui fait cruellement défaut aux quartiers centraux du Caire. 
Illustration 3 - Carte du changement social en Égypte

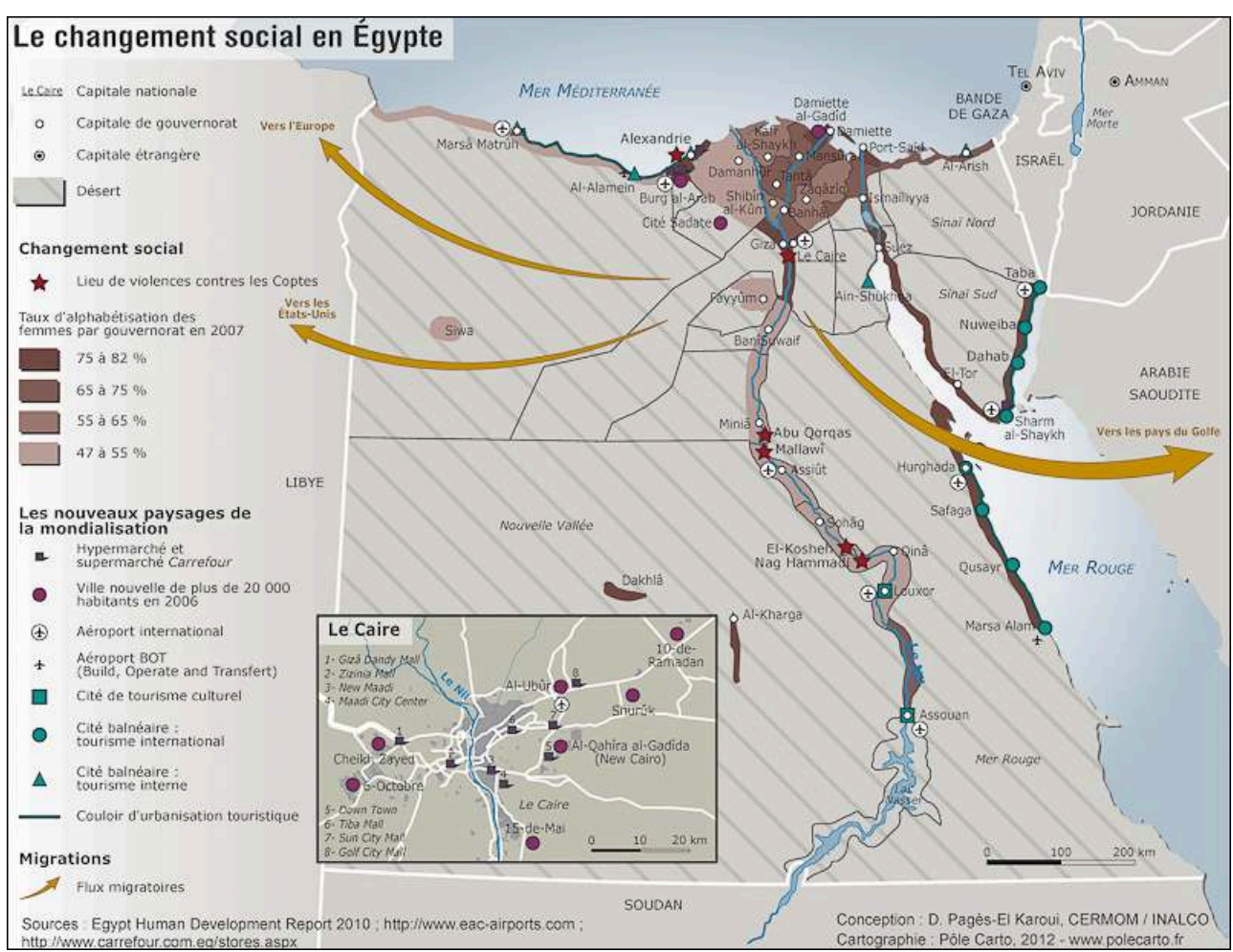

L'outil de séduction des classes aisées a été les gated communities (compound en égyptien). Ce sont des ensembles résidentiels qui généralement mêlent habitat individuel et collectif, de taille très variable. Construits par des promoteurs privés, ils sont protégés par une enceinte et un système de surveillance, et dotés de leurs propres équipements et services (ramassage des ordures, eau et assainissement, système de transport, supermarchés, écoles...). Pour les plus riches, les gated communities offrent un nouveau modèle urbain qui privilégie l'entre-soi et renoue avec un mode de logement qui avait disparu, - tant la pression foncière était forte -, la villa. Avec le succès des gated communities, les services ont suivi dans les villes nouvelles et alors que ces cités construites en plein désert, souffraient d'un déficit de centralité, on a vu se multiplier les ouvertures d'écoles, d'universités et d'hôpitaux privés, et l'arrivée de petits commerces de proximité ou de grandes surfaces.

Les malls, ces gigantesques centres commerciaux dont les bâtiments sont souvent adossés à d'autres activités (hôtels internationaux, appartements luxueux, bureaux de standing, restaurants et cinémas) constituent un autre symbole de cette nouvelle urbanité. Le Caire en compte une trentaine, situés à proximité des quartiers résidentiels aisés, des centres d'affaires ou des grands centres touristiques. Ces nouveaux temples de la consommation qui offrent aussi bien des marchandises de marques internationales, que des produits artisanaux locaux ou fabriqués à la chaîne en Chine, ne sont pas seulement fréquentés par les gens qui ont les moyens de s'acheter ces produits, mais aussi par des flâneurs qui viennent s'y promener ou faire du lèchevitrines. Les malls se sont donc rapidement imposés comme des nouveaux lieux de sociabilité tant pour les jeunes que pour les familles, dans une ville où les lieux de rencontre ne sont pas si nombreux. Mais, parce que l'entrée de ces malls est filtrée par un service de sécurité, il est difficile de parler d'espaces publics. 
Illustration 4 - Le centre commercial City Star au Caire

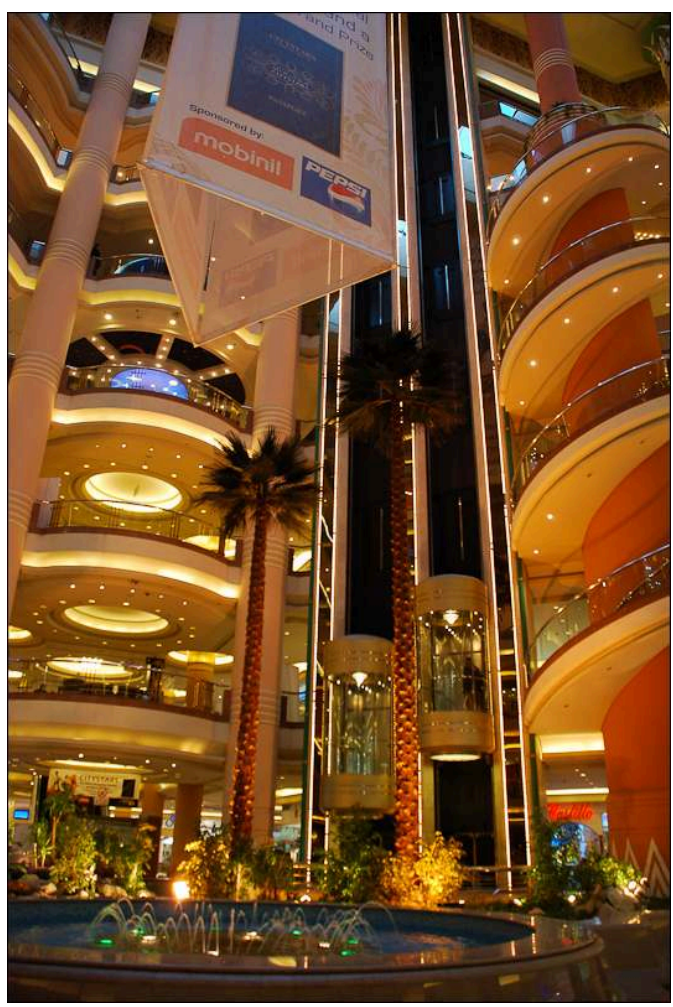

Auteur : D. Pagès-El Karoui, mars 2008.

Illustration5 - Publicité pour le projet immobilier Le Rêve (ville nouvelle de New Cairo)

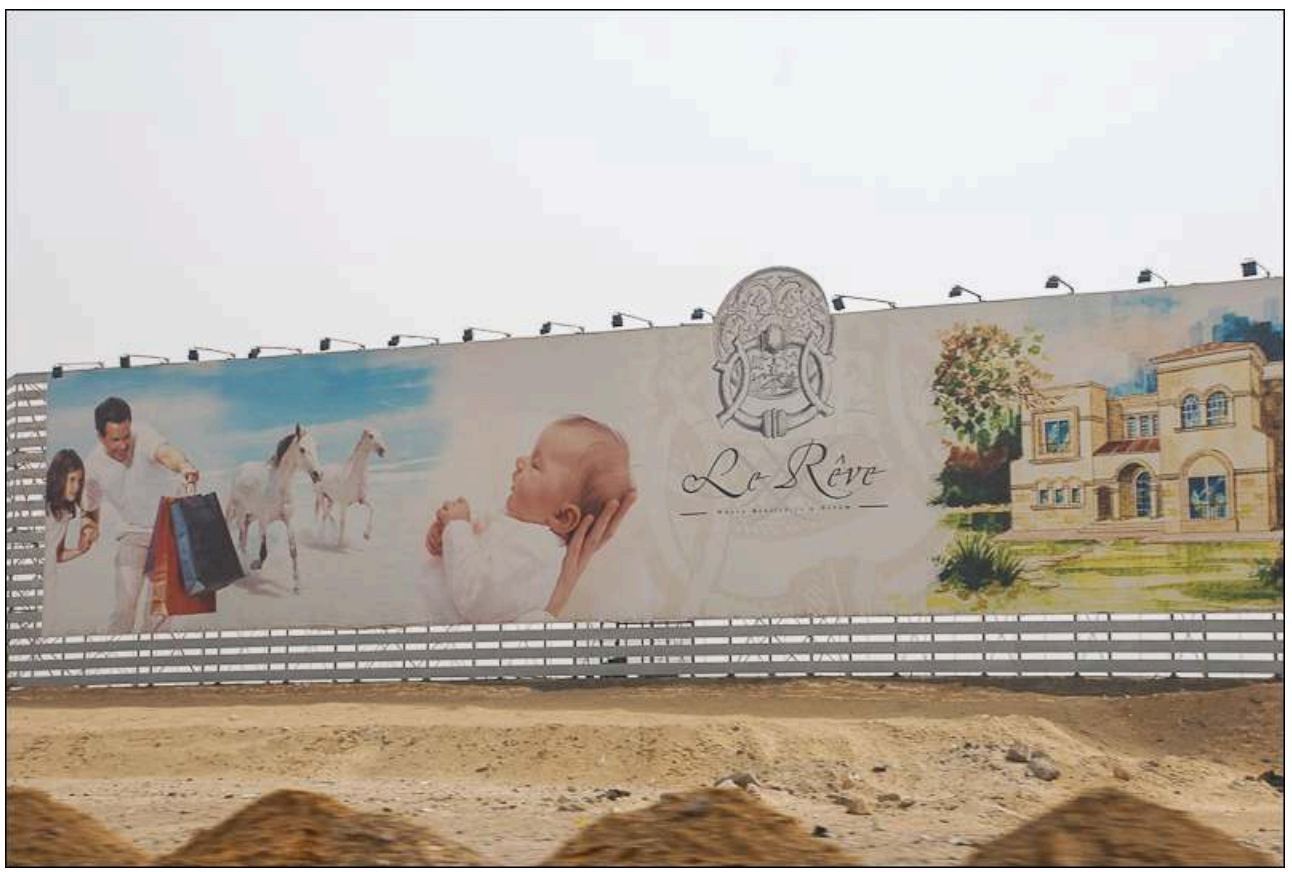

Auteur : D. Pagès-El Karoui, mars 2008. 
Illustrations 6- Rehab, une gated community du Grand Caire

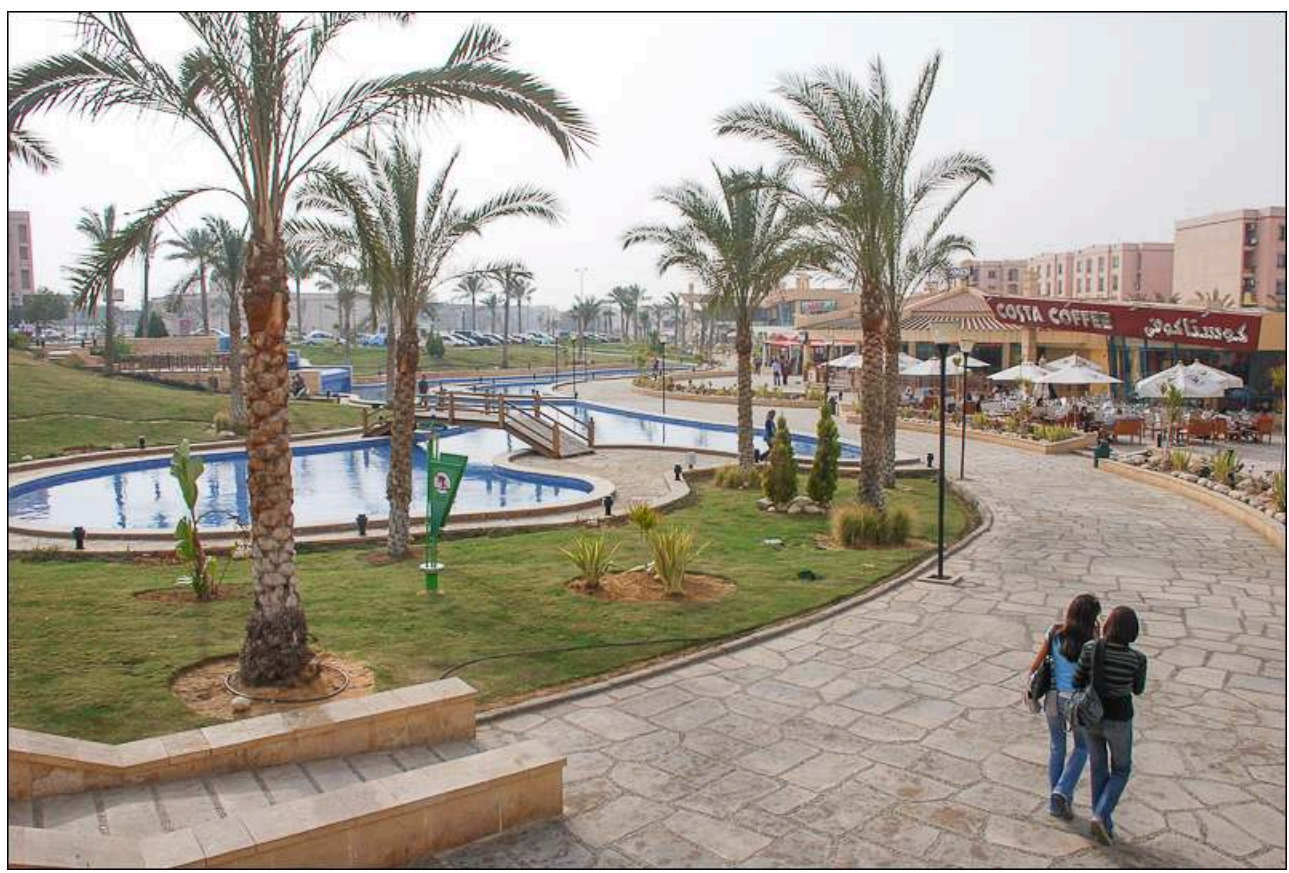

Le centre ville de Rehab

Auteur : D. Pagès-El Karoui, mars 2008.

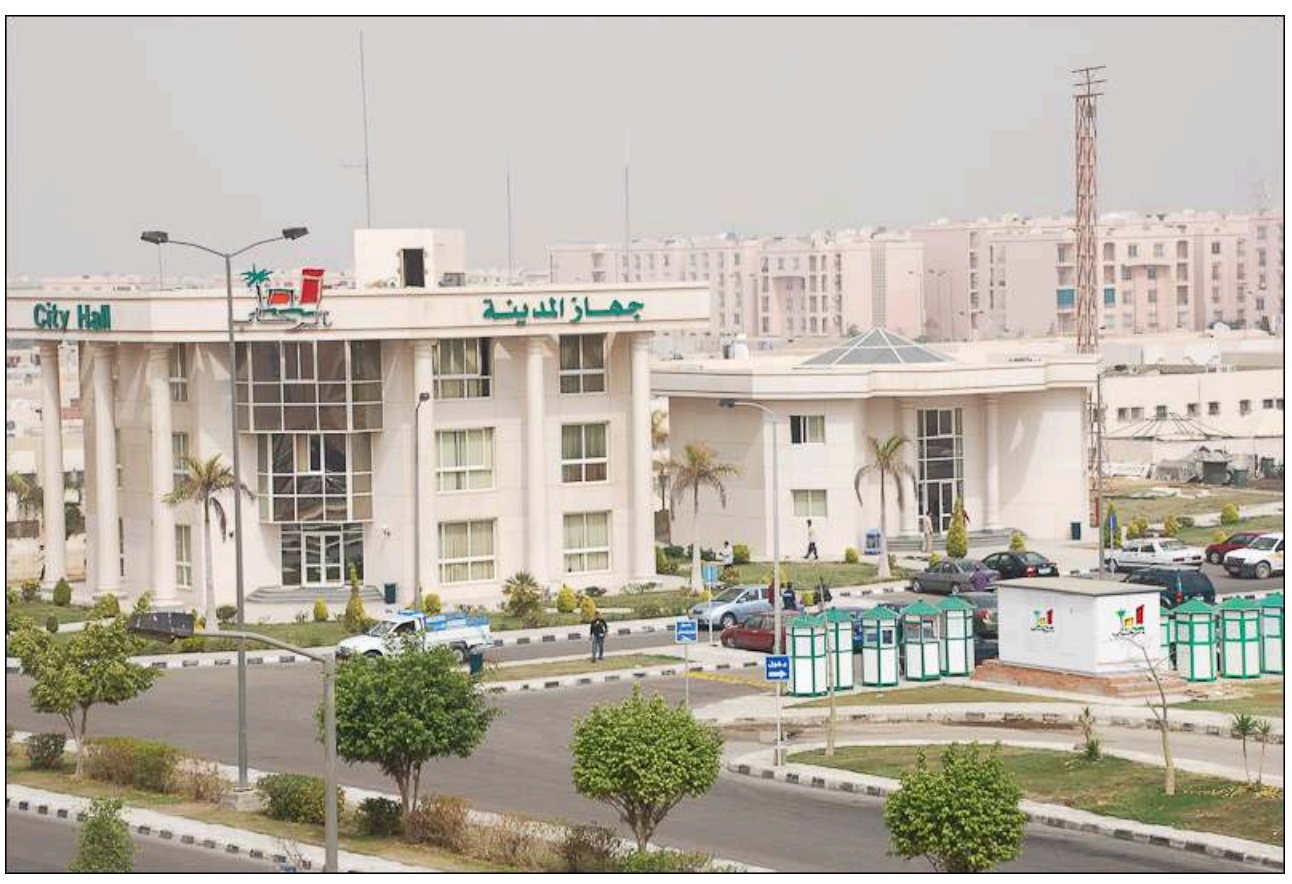

La « mairie » de Rehab

Auteur : D. Pagès-El Karoui, mars 2008. 


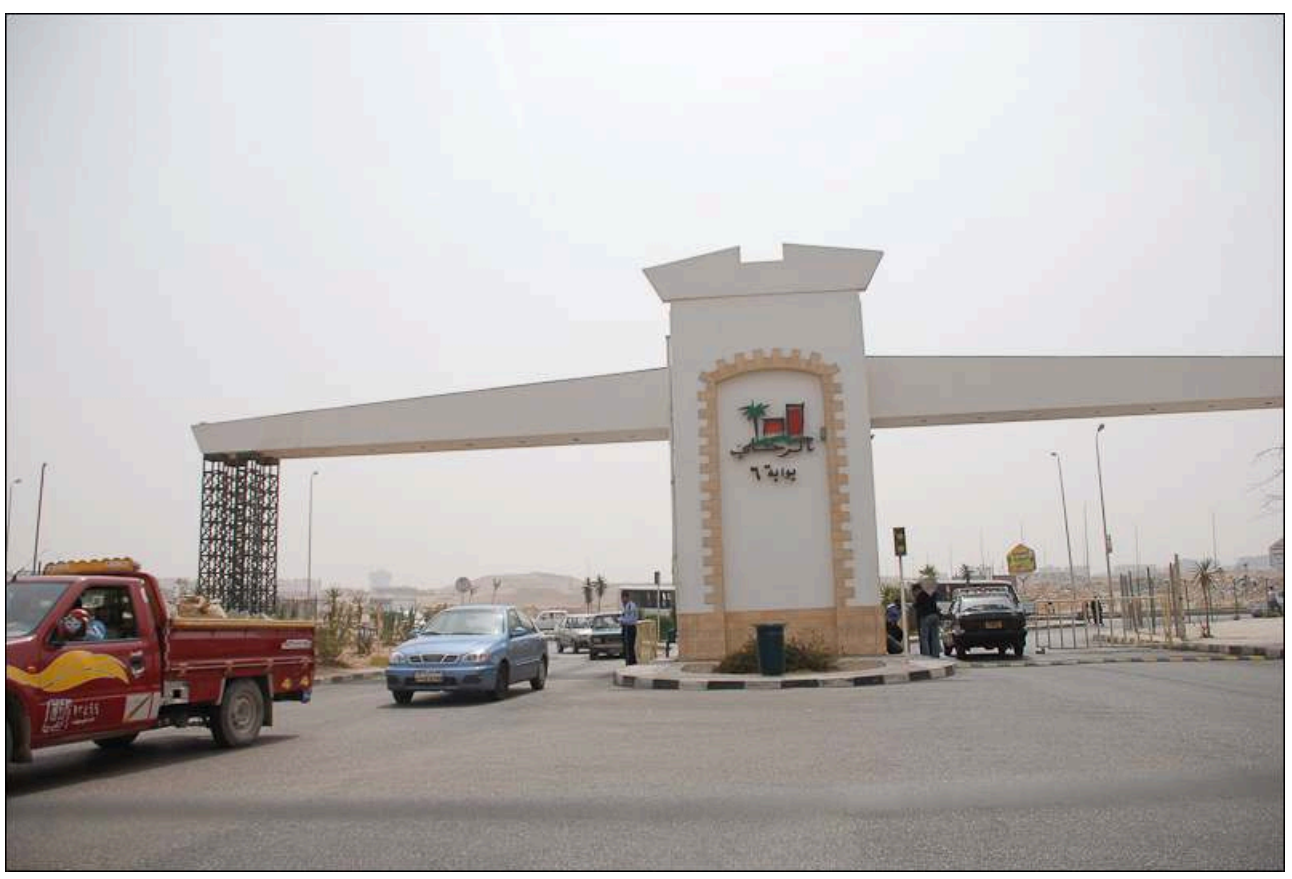

Une entrée de Rehab

Auteur : D. Pagès-El Karoui, mars 2008.

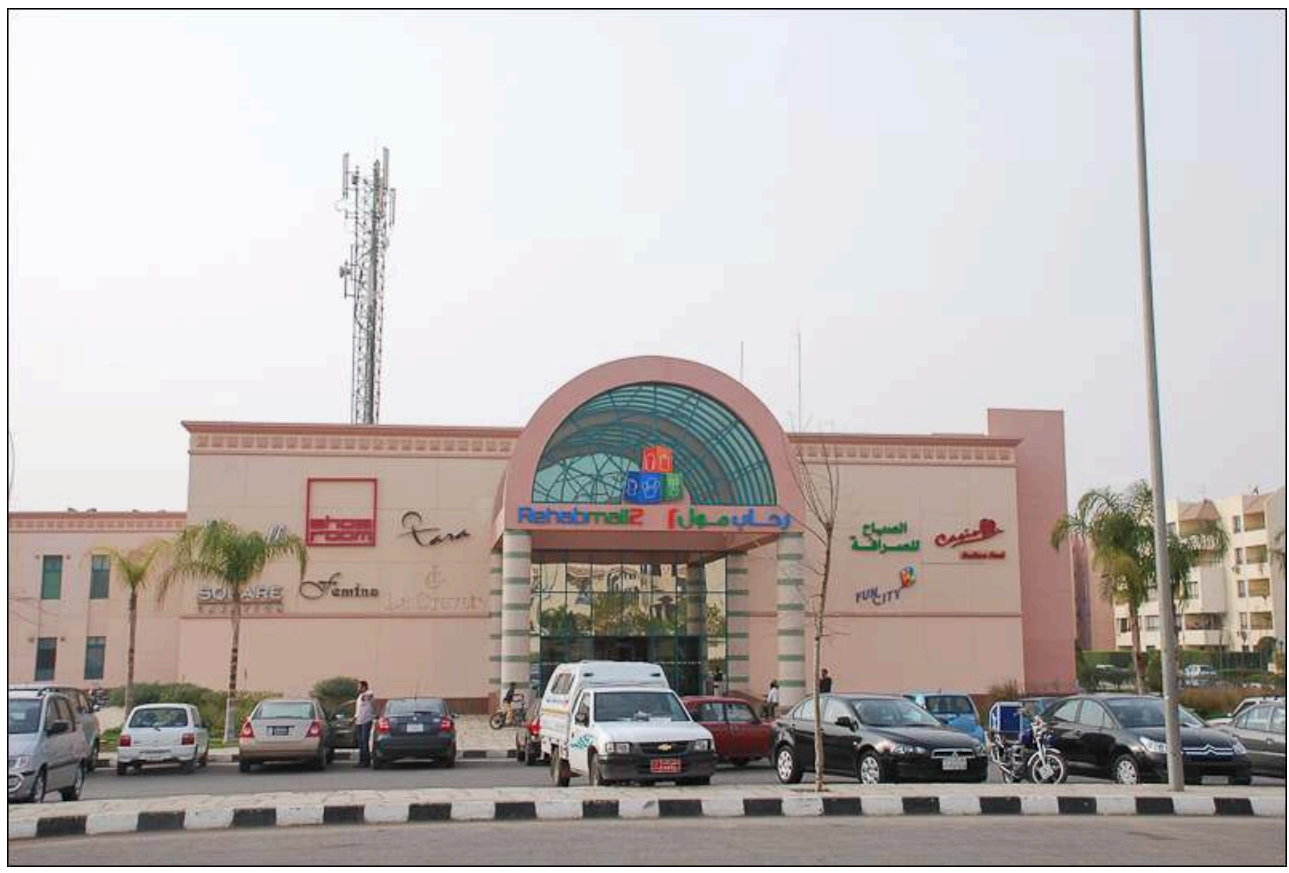

Un mall de Rehab

Auteur : D. Pagès-El Karoui, mars 2008.

41 Ces gigantesques malls et luxueuses cités résidentielles fermées ont souvent été interprétés comme des signes de la privatisation de la ville et comme le profond révélateur d'inégalités sociales croissantes. Ces nouveaux paysages urbains du GrandCaire expriment cette influence des modèles urbains états-uniens, qui ont transité par les villes du Golfe, elles-mêmes fortement influencées par Hong-Kong et Singapour. La circulation des modèles est telle que le terme d'américanisation ou d'occidentalisation ne paraît plus si pertinent. Le développement de cette société de consommation 
provoque de nouvelles stratégies de distinction et pousse, dans une dynamique de fragmentation de la société, les classes urbaines les plus aisées à s'exiler loin du centre et à s'isoler de la masse compacte, bruyante et dégradée de la ville ancienne. Pourtant, le modèle des villes nouvelles a ses limites: d'une part, à cause de leurs verdoyants jardins, elles sont fortement consommatrices d'eau, et d'autre part, elles ne font finalement qu'étendre considérablement la superficie de la ville et renforcer la polarisation autour du Caire. Alors que le taux de motorisation est encore bas en Égypte, (43 véhicules pour 1000 habitants en 2008, contre 600 en France ${ }^{15}$ ), la vente de voitures a très fortement augmenté ces dernières années, rendant la circulation infernale dans les grandes villes, augmentant la pollution, les embouteillages n'épargnant pas les villes nouvelles.

Pendant la révolution, ces lieux de la mondialisation ont pu être pris pour cible : le Carrefour de Maadi a été pillé, des malls ont été saccagés. Les villes nouvelles n'ont pas été des grands lieux de la contestation populaire: leurs habitants ont préféré se déplacer à Tahrir pour aller manifester, ce qui peut être interprété comme un échec à créer de la centralité symbolique.

\section{Les nouveaux espaces du tourisme}

43 L'essor du tourisme international (les touristes étaient 2 millions en 1990 et 15 millions, 20 ans plus tard), a contribué au remodelage des territoires (cf. illustration 3). La géographie touristique s'est trouvée totalement modifiée avec la diversification du tourisme égyptien, autrefois essentiellement culturel, lié aux vestiges pharaoniques de la vallée du Nil, et aujourd'hui de plus en plus balnéaire. Les littoraux désertiques de la Mer Rouge et du Sinaï qui abritent actuellement $62 \%$ des hôtels égyptiens (Vignal, 2010), ont été profondément urbanisés, suite à un boom de la construction hôtelière et résidentielle (vente de résidences secondaires pour des Égyptiens ou à des étrangers du Golfe). Cette urbanisation se traduit par la croissance de cités balnéaires, comme ElArish, Sharm al-Shaykh, Hurghada, qui, de plus, disposent de fonctions administratives en tant que capitales de gouvernorats, et sont bien desservies par des aéroports internationaux actifs. De taille plus réduite, les villages touristiques intégrés (resorts) se multiplient. Il s'agit d'enclaves hyperspécialisées, totalement déconnectées de la réalité du pays, qui fonctionnent grâce à une main d'œuvre temporaire, masculine, venue du Delta et de la Vallée. Le modèle est assez proche des gated communities, puisque les resorts disposent généralement de leurs propres infrastructures et services (usine de dessalement de l'eau de mer, station de traitement des eaux, centrale électrique) et relèvent aussi d'une privatisation des espaces et des infrastructures.

Ces resorts sont financés par des capitaux égyptiens (le grand groupe Orascom a son resort al-Gouna au nord d'Hurghada) ou du Golfe. Le resort de Port-Ghalib qui appartient au groupe koweitien Kharafi est géré par une société sud-africaine. Ouvert en 2007, il s'étend sur un ruban de $18 \mathrm{~km}$ de long et regroupe des hôtels de luxe, des résidences de standing, un grand centre de conférence, un golf, une marina. Une lagune artificielle a été creusée et un souk égyptien traditionnel reconstitué. Situé à $250 \mathrm{~km}$ au sud d'Hurghada, Port-Ghalib est accessible par Marsa Alam, premier aéroport privé d'Égypte, construit en système BOT (Build, Operate and Transfer) par le même groupe Kharafi, et dont la gestion a été confiée à la société ADP (Aéroports de Paris). L'aéroport offre un accès direct à cette nouvelle destination de la mer Rouge, plus préservée et 
plus proche de la vallée, qui permet de jouer sur la complémentarité de séjours balnéaires et culturels (Sanmartin, 2011).

Avec l'essor du tourisme national, ces espaces sont de plus en plus fréquentés par les Égyptiens des classes moyennes et supérieures. C'est d'ailleurs à l'intention spécifique de cette clientèle nationale, que s'est faite l'urbanisation de la côte méditerranéenne (la côte nord, comme l'appellent les Égyptiens) qui égrène, entre Alexandrie et Marsa Matrûh, sur $300 \mathrm{~km}$, plus de 120 villages touristiques, offrant plusieurs types de résidences d'été ( $\mathrm{du}$ petit appartement à la grande villa avec jardin et piscine), totalement désertées en dehors de la période estivale. On voit même se développer chez les gens les plus aisés, l'achat de deux résidences secondaires, l'une sur la côte nord pour l'été, et l'autre sur la Mer Rouge à Ain Sokhna, par exemple, pour les week-ends d'hiver.

Ce secteur fondamental pour l'économie égyptienne (13\% de la population active et $11 \%$ du PIB) a été malmené par les répercussions de la révolution: le nombre de touristes a chuté de $50 \%$ en 2011, de nombreux employés sont au chômage technique, les grands sites sont désertés (le tourisme balnéaire s'en sort mieux) et les réserves de devises ont chuté. Le tourisme résume à lui seul les incertitudes économiques qui pèsent actuellement sur l'Égypte.

\section{L'urbanisation des campagnes}

Illustration 7 - Minyat Sandûb, un « village » de l'agglomération de Mansûra (delta du Nil)

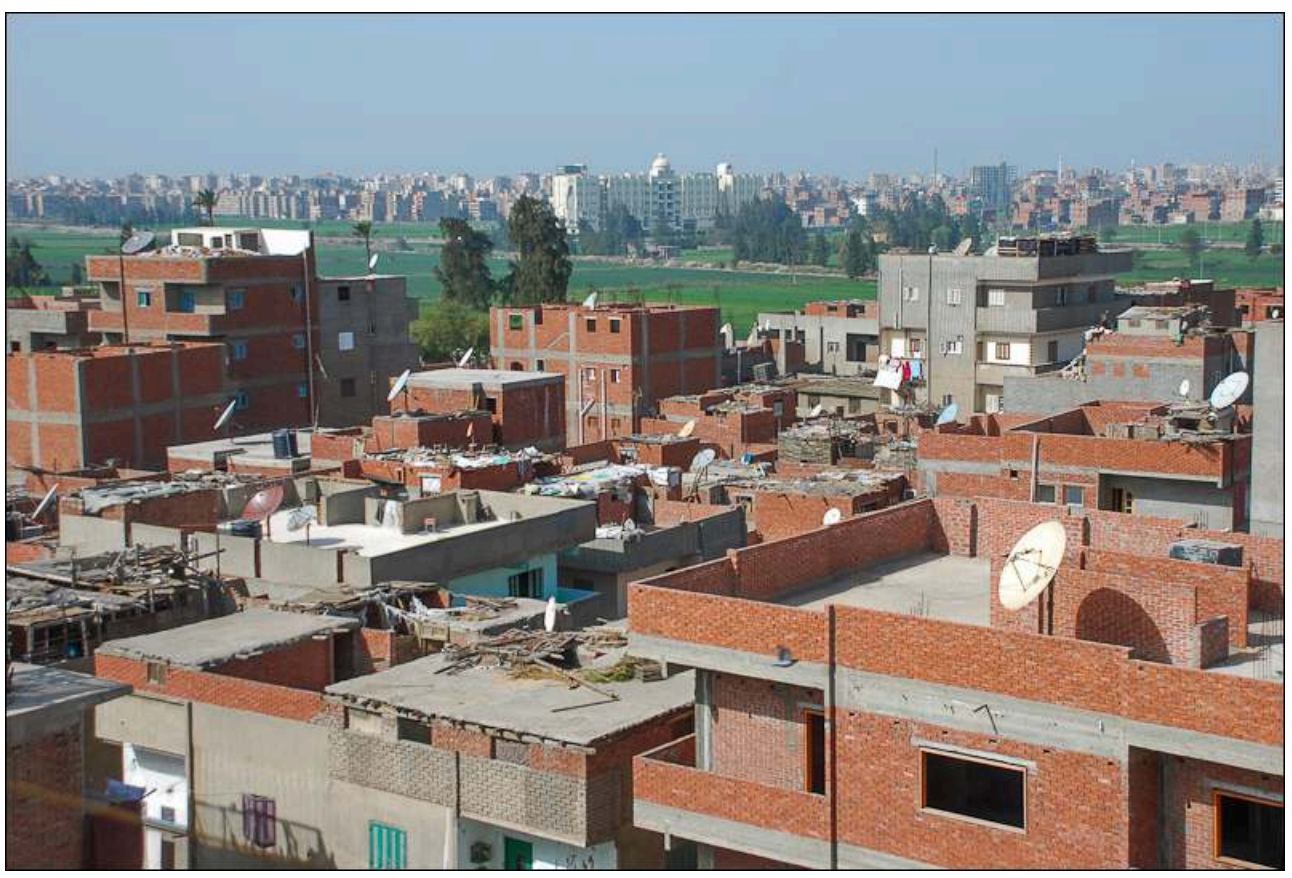

Auteur : D. Pagès-El Karoui, mars 2008

Cette urbanisation des campagnes se manifeste notamment par une transformation radicale de l'habitat (cf. illustration 7). L'ancienne maison centrée sur une cour intérieure qui était le domaine de la famille élargie, a été remplacée par l'immeuble de 3-4 étages, en armature de béton et murs de briques - comme dans les quartiers informels -, qui est généralement la propriété d'une famille, chaque étage étant dévolu 
à l'appartement d'une famille nucléaire (les grands-parents au rez-de-chaussée quand ils sont encore en vie, les parents au premier étage, et les enfants mariés ensuite, généralement les fils). Il s'agit là d'un compromis entre famille élargie et famille nucléaire : les parents et leurs enfants habitent les uns près des autres mais chacun dans son appartement.

Il arrive aussi de plus en plus que les enfants n'habitent pas sur place. Une importante offre locative s'est donc développée dans ces villages qui attirent des ménages modestes, chassés par les prix très élevés des loyers en ville. Avec le déclin de la fonction agricole (il est bien plus rentable pour un paysan de vendre sa terre pour qu'elle soit bâtie que de continuer à la cultiver), les activités se diversifient dans les villages où s'ouvrent de nombreuses épiceries, pharmacies, boutiques de vêtement, salons de coiffure et ateliers de réparation mécanique... Face à cela, le niveau d'équipement (eau potable, assainissement, routes goudronnées, éclairage public) reste très variable d'un village à un autre.

Mais la transformation n'est pas que matérielle, ce sont aussi les modes de vie qui ont été chamboulés par la montée de l'individualisme. Les jeunes femmes éduquées, souvent diplômées de l'université, adoptent un style de vie urbain : elles s'habillent selon la mode citadine, elles ne respectent plus le mythe de l'auto-consommation (elles achètent leur pain à la boulangerie et ne le font plus elles-mêmes), elles réclament à leur mari l'équipement ménager le plus moderne (gazinière, machine à laver...) et pardessus tout, elles souhaitent s'émanciper de la tutelle de leur belle-famille, puisque que traditionnellement, la jeune mariée venait vivre auprès des parents de son époux, dans le cadre de la famille élargie. Elles sont de plus en plus nombreuses à réclamer un espace à elles. Cette promotion de la famille nucléaire est mal perçue par les générations plus anciennes qui ont le sentiment de ne plus voir suffisamment leurs enfants, qui seraient devenus des étrangers à leur propre famille. Alors que le village était le lieu par excellence de l'interconnaissance, l'anonymat se répand de plus en plus dans les bourgs.

50 C'est que les campagnes, à l'image du pays tout entier, sont prises dans une très forte mobilité : un dense réseau de transports en commun (microbus, touks-touks ${ }^{16}$ ) irrigue tout le territoire jusqu'aux villages plus reculés. Au village, cette mobilité est double. Elle se manifeste tant par des flux de départ, ou des circulations incessantes, de ses habitants vers la ville secondaire la plus proche, vers la capitale ou vers l'étranger, que par des flux d'arrivée de ces anciens citadins qui viennent s'installer au bourg, sous la contrainte économique. Mais cette intense mobilité ne doit pas faire oublier la forte inactivité qui touche souvent les habitants. Le chômage étant endémique, surtout pour les jeunes, les villages ont vu se multiplier les cafés où se réunissent les hommes inactifs, qui passent leurs journées à discuter et à jouer, tandis que les jeunes filles restent à la maison, rêvant à un avenir meilleur devant leurs écrans de télévision ou d'ordinateur.

51 Ces nouveaux paysages, dont nous avons livré ici quelques exemples, sont révélateurs de l'amplitude des transformations sociales, dont certaines sont imputées à l'essor des migrations vers le Golfe. 


\section{Migrations et changement social}

52 L'Égypte est devenue l'un des plus grands pays d'émigration de la région du MoyenOrient, avec environ 6 millions d'Égyptiens expatriés surtout dans le Golfe, mais aussi en Amérique du Nord et en Europe. Encouragée par la libéralisation (infitâh) de Sadate dans les années 1970, l'émigration économique temporaire constitue depuis une soupape de sécurité pour le pouvoir, face aux blocages économiques, politiques et sociaux du pays. Mais, c'est aussi une ressource financière avec les transferts d'épargne des émigrés devenus essentiels pour le développement économique du pays. Ainsi, face à l'incapacité des nouvelles stratégies économiques à créer suffisamment d'emplois pour absorber les jeunes arrivants sur le marché du travail, l'expatriation est devenue pour un nombre croissant d'Égyptiens une façon privilégiée, quoique risquée car de plus en plus souvent illégale (Zohry, 2007), d'échapper au déclassement social. Dans quelle mesure les migrants participent-ils - à leur retour, de loin, ou via leurs proches restés au pays -, au changement social en cours en Égypte à travers les idées, valeurs et pratiques auxquelles ils ont été confrontés lors de leur expérience migratoire - ce que Peggy Levitt (1998) a nommé social remittances - et qu'ils réadaptent chez eux? Les migrants sont-ils des accélérateurs ou au contraire des freins au changement social ${ }^{17}$

\section{Débats sur l'impact des migrations sur la démographie}

53 L'impact des migrations sur les comportements démographiques a suscité de larges débats en Égypte (Hoodfar, 1997 ; Louhichi 1997). L'hypothèse la plus courante défend que les migrations font reculer la fécondité, en retardant l'âge du mariage (ce sont en majorité des hommes seuls qui émigrent avant leur mariage ou avant la naissance de leurs enfants), en contribuant à l'émancipation des femmes, ainsi qu'en favorisant la promotion de la famille nucléaire, via la décohabitation des ménages grâce aux transferts d'épargne (cf. supra). D'autres chercheurs insistent au contraire sur le fait que les femmes restées seules sans leur mari sont souvent sous la coupe de leur bellefamille et que l'amélioration des conditions de vie des migrants contribue à perpétuer les valeurs conservatrices : par exemple, quitter son travail pour une femme de migrant peut être perçu comme un signe de promotion sociale.

En Égypte, la migration apparaît souvent comme une affaire masculine, car il reste du ressort de l'homme de subvenir aux besoins de la famille, même si le travail féminin se développe rapidement avec la crise. Cependant, les femmes sont tout de même un certain nombre à partir, soit dans le cadre du regroupement familial (surtout dans le Golfe pour les classes moyennes et supérieures), soit pour aller elles-mêmes travailler. Quand les femmes partent seules, le contrôle exercé sur elles par les familles est très fort. Quand la femme est mariée, l'inversion des rôles est souvent mal vécue par le mari et cela peut parfois conduire au divorce. Quant à celles qui sont restées seules en Égypte, les résultats des enquêtes ethnographiques sont très ambivalents. Dans certains cas, le contrôle exercé par la belle-famille peut être pire que celui du mari, d'autres connaissent une émancipation temporaire, qui disparait avec le retour de leur conjoint, tandis que quelques unes vivent une réelle amélioration de leur statut.

55 L'impact des migrations sur la fécondité varie aussi selon les pays de destination. À partir d'une étude quantitative sur les taux de fécondité, Philippe Fargues (2006) a démontré un impact différencié des migrations sur la démographie en fonction des 
pays d'accueil. Il explique des taux de fécondité plus bas au Maghreb et en Turquie qu'en Égypte (3 enfants par femme) par des systèmes migratoires davantage tournés vers l'Europe pour les premiers et vers le Golfe pour le second. En Égypte, les remises sociales venant de sociétés plus conservatrices et patriarcales auraient freiné la transition démographique, tandis que celles venues des sociétés plus libérales l'auraient accélérée au Maghreb et en Turquie. À l'échelle micro, des enquêtes ethnographiques suggèrent des résultats différents. Elles démontrent que cette opposition entre sociétés libérales et conservatrices est souvent instrumentalisée par les migrants pour célébrer les normes familiales égyptiennes, comme un entre-deux entre un système jugé trop conservateur, celui du Golfe et un autre trop libéral, celui de l'Occident (Gruntz, Pagès-El Karoui, 2012).

\section{Quand la migration encourage l'urbanisation}

56 Si l'articulation entre migration et urbanisation a suscité des travaux novateurs en Afrique subsaharienne (Tall, 2009; Bertoncello, 2010), elle a été beaucoup moins analysée en Égypte, où les études ont surtout porté sur l'urbanisation des villages (Muller-Mahn, 1998). Là-bas, comme ailleurs, l'achat ou la construction d'un logement est la façon la plus courante d'investir l'argent mis de côté et de manifester sa réussite sociale. Grâce à l'épargne accumulée, les migrants ruraux ont massivement acheté des terres agricoles pour construire des immeubles. Ils sont tenus pour responsables de la formidable extension des villages et de la forte inflation des prix du foncier. Dans ces bourgs, certains quartiers périphériques sont appelés « quartiers des émigrés ». Ce sont eux aussi qui sont généralement les promoteurs de ce nouvel élément architectural de l'espace rural que sont les «tours » de plus de dix étages qui nécessitent l'installation d'un ascenseur (voir illustration 8). Autre innovation qui est souvent attribuée aux migrants, le fait de louer un appartement dans l'immeuble familial. Ce nouvel usage rompt avec la perception de l'espace habité, comme un espace privé, réservé aux membres de la famille. Contrairement aux processus de privatisation de la ville que l'on a décrit plus haut, il y a là un processus inverse de glissement du privé vers le public, aux moins pour les parties communes de l'immeuble, que l'on est prêt à partager avec des inconnus, ce qui était peu envisageable il y a quelques décennies.

Les migrants participent aussi à l'essor des mobilités, car nombre d'entre eux ont de retour du Golfe, acheté un microbus ou un touk-touk et se sont transformés en autoentrepreneurs, assurant soit les liaisons internes au bourg, rendues nécessaires par son extension récente, soit les liaisons avec la ville secondaire la plus proche. En revanche, on en sait beaucoup moins sur les pratiques des migrants urbains, mais les premières impressions glanées sur le terrain suggèrent qu'ils sont aussi des acteurs majeurs de la production urbaine. Il est clair que les migrants constituent une clientèle privilégiée pour l'achat des résidences des resorts ou des gated communities. Leur séjour à l'étranger les a habitués à certains modes de vie qu'ils souhaitent retrouver à leur retour. De nombreux cafés "branchés " pour une clientèle mixte, les coffee shops, ont ouverts au Caire : leurs patrons sont souvent d'anciens expatriés aux États-Unis ou en Europe, de retour au pays (De Koning, 2006). 
Illustration 8 - Immeuble construit par un émigré en Italie (village de Tatûn, Fayyûm)

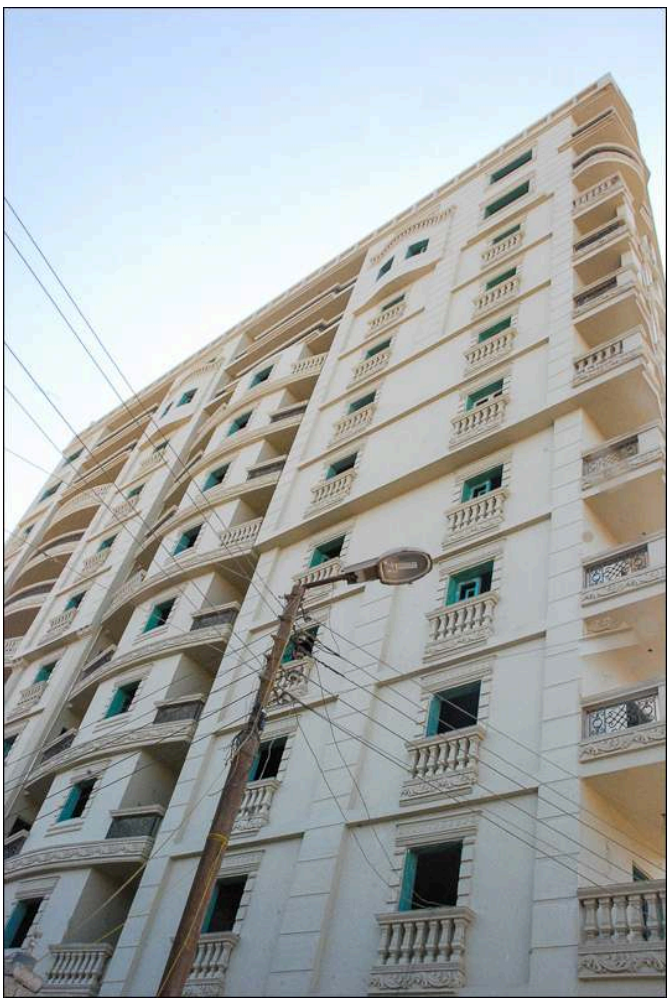

Auteur : Pagès-El Karoui, sept. 2011 (ANR SYSREMO)

Illustration 9 -Un touk-touk appartenant à un migrant d'Italie (village de Tatûn, Fayyûm)

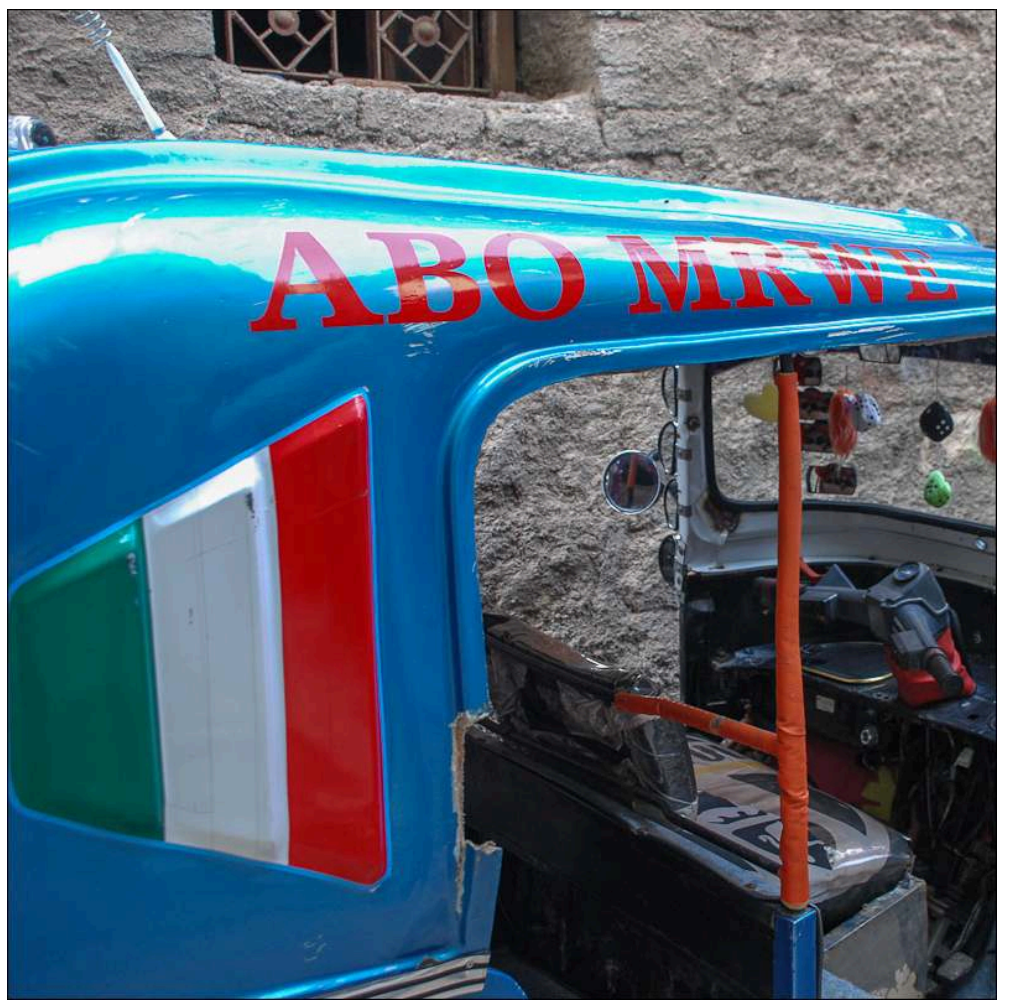

Auteur : Pagès-El Karoui, sept. 2011 (ANR SYSREMO). 


\section{Les migrants, un péril pour l'ordre social ?} consommation ostentatoires importés du Golfe, ont souvent été décriés par les intellectuels, journalistes, romanciers ou cinéastes. Selon eux, la migration serait à elle seule le symbole de tous les maux de l'infitâh, associée à la désintégration de la société (tafasukh), à la culture de la consommation et du gaspillage (istihlâk), et aux dangers de la perte identitaire. Les migrants deviennent les boucs émissaires privilégiés pour dénoncer le changement social. Familiarisés avec la société de consommation dans le Golfe, les migrants égyptiens, de retour chez eux, provisoirement ou définitivement, s'en font les vecteurs. Ils reviennent les bras chargés de paquets (vêtements, jouets, électroménager, couverture, ventilateur, lecteurs DVD...) pour eux ou pour leurs proches. Ils dénoncent fréquemment un sentiment de prédation dont ils se sentent victimes de la part de ceux qui sont restés, qui attendent avidement des cadeaux, manifestant la réussite de l'expatriation. le Golfe, rendus responsables de l'importation d'un islam wahhabite, conservateur et rigoriste, venu d'Arabie Saoudite, différent des pratiques religieuses égyptiennes. Cette explication, si elle n'est pas suffisante pour éclairer à elle seule la réislamisation de l'Égypte - l'islamisme n'est pas exogène et les gouvernants, égyptiens comme saoudiens, y sont aussi pour beaucoup - rappelle que, si en Europe, les migrants ont tendance à être souvent perçus comme une menace pour l'identité des sociétés d'accueil, ils peuvent aussi constituer, dans les sociétés de départ, un péril pour l'ordre social, via les idées, valeurs et pratiques qu'ils rapportent et réinterprètent.

En réalité, ces «transferts sociaux » varient considérablement en fonction du capital social et des compétences individuelles du migrant, du poids des contraintes familiales et collectives imposées par son milieu d'origine, de l'importance des réseaux transnationaux qu'il a pu tisser, de la qualité de son insertion dans la société d'accueil, et enfin des politiques migratoires en Égypte et dans les pays d'accueil. Il résulte de cette combinaison toute une multitude de scénarios possibles qui vont du métissage à la résistance à l'hybridation. Si l'émigration concourt à l'individualisation de la société et favorise la famille nucléaire, via l'achat d'un logement indépendant, les migrants restent généralement exposés à la pression familiale et à un sévère contrôle social qui s'exerce à la fois à distance et au sein des communautés expatriées. Dans certains cas, les remises sociales peuvent être très limitées : de nombreux Égyptiens d'origine rurale ou périurbaine, vivent illégalement en France de nombreuses années, en ne parlant qu'égyptien et en ayant une sociabilité uniquement réduite à la communauté égyptienne dont les réseaux leur procurent travail et logement (Müller-Mahn, 2005).

61 Ainsi les migrations contribuent aux changements sociaux, mais dans un sens largement équivoque, et pas forcément révolutionnaire. Le cas égyptien permet de confirmer l'analyse de Jean-François Bayart et Fariba Adelkhah (2007,p. 11): via leurs remises sociales, les migrants «exercent une influence importante sur l'évolution de leur société d'origine sans que l'on puisse attribuer à celle-ci une orientation, de type «progressiste » ou «conservatrices » : pour le meilleur ou pour le pire, ils constituent un levier du changement social et un ferment de l'idée nationale : ils participent aussi du jeu politique factionnel et aux fondements sociaux de l'État: ils introduisent un 
tiers-principe dans le processus de production des statuts en faisant que de la distinction sociale se forme désormais de plus en plus off-shore ».

Il est encore trop tôt pour se prononcer sur l'impact de la révolution sur les migrations. Au-delà des craintes de l'UE d'une « invasion » de migrants suite au printemps arabe, il semble que pour l'Égypte le champ migratoire ne se soit pas radicalement transformé : une enquête menée par l'Organisation Internationale des Migrations (IOM, 2011) démontre que le taux de candidats potentiels à l'émigration chez les jeunes est stable par rapport à 2009, autour de $15 \%$. Certes, une petite minorité de migrants qualifiés sont rentrés au pays, pour soutenir le soulèvement ou le processus de transition en cours - Waël Ghoneim, directeur marketing Moyen-Orient de Google à Dubaï en est l'exemple le plus célèbre -. Par leur engagement direct ou par leur soutien financier ${ }^{18}$, les émigrés ont joué un rôle important dans le processus révolutionnaire. Les Égyptiens de l'étranger ont d'ailleurs obtenu de voter pour la première fois aux élections de leur pays en 2011. D'autres, au contraire, quittent l'Égypte car ils redoutent la longueur de la transition ou l'établissement d'un régime islamiste: une ONG annonce le départ de 100000 coptes, sans que la source de ces chiffres soit clairement établie. En octobre 2011, la Banque centrale égyptienne annonçait un record atteint par les transferts d'épargne (12,6 milliards de \$). Ce chiffre a été interprété de façon contradictoire, soit comme un signe de solidarité des migrants envers les difficultés rencontrées par leurs familles sur place, soit comme la traduction du rapatriement des fonds des Égyptiens de retour des pays arabes, notamment de Libye (Abdelfattah, 2011).

\section{Conclusion}

63 À travers cette analyse, nous avons souhaité démontrer la force des changements sociaux en amont de la révolution du 25 janvier 2011, et leurs traductions spatiales, bien loin du paradigme de l'immobilité, celui de l'Égypte éternelle. Ces changements qui parcourent l'ensemble du monde arabe éclairent et rendent compréhensibles les «miracles» du printemps arabe. Bien entendu, ces changements ne touchent pas l'Égypte de manière homogène. De plus, ces processus sont ambivalents, on a vu tout le poids du conservatisme et du contrôle social, ce qui laisse penser que la transition démocratique sera longue et incertaine. Quelle sera la position du gouvernement islamiste face aux transformations sociales? Pour être complète, cette réflexion devra être prolongée par une analyse détaillée du soulèvement du 25 janvier comme accélérateur du changement social. Mais le recul manque et les sources sont trop lacunaires ou au contraire trop vastes pour que les chercheurs soient en mesure de la mener à bien dès à présent.

Si la révolution sociale n'était pas au programme des révolutionnaires et si la révolution politique est loin d'être aboutie (le président Morsi envoie des signaux contradictoires d'affranchissement vis-à-vis des militaires et de maintien de la censure des médias), la révolution la plus tangible est celle des mentalités. Une dynamique culturelle profonde s'est enclenchée dans la société, qui ne touche pas seulement la petite minorité des manifestants de la place Tahrir : la disparition du mur de la peur, la possibilité de renverser collectivement le tyran, d'être maître de son destin. L'émancipation de la parole politique est impressionnante, Stefano Savona l'a bien montré dans son film Tahrir, place de la Libération. Aujourd'hui, à la télévision, les 
séances du parlement sont suivies avec autant d'intérêt, si ce n'est plus, que les films et les talk-shows.

Dans cet article, nous avons beaucoup insisté sur les processus structurels (urbanisation, migration, transition démographique) et globalisés (libéralisation, essor de la société de consommation, ouverture au monde via les nouvelles technologies) qui sont producteurs de changement. Inspirée par la démarche d'Asef Bayat (2009), une autre approche aurait pu aussi davantage souligner les changements sociaux impulsés par le bas. Bayat distingue les pratiques extraordinaires des activistes, dont il met en exergue les échecs (la révolution de 2011 témoignera cependant de leur renouveau et de leur efficacité), de ce qu'il appelle la « citoyenneté active (active citizenry) » et qui a joué un rôle crucial dans la transformation des sociétés moyen-orientales. Ce concept renvoie aux pratiques quotidiennes d'acteurs ordinaires (habitants des quartiers informels, vendeurs de rue, migrants ruraux...), qui par leurs «empiètements silencieux du quotidien », en dehors de toute idéologie mais poussés par la nécessité, ont investi par leur " art de la présence » de nouveaux espaces dans lesquels ils ont réussi à se réapproprier des biens sociaux, à se faire entendre, et à acquérir une certaine autonomie. La multiplication des conflits entre ces citoyens les plus pauvres et l'État a conduit à de nombreuses occupations de l'espace public. C'est de ce terreau des lents apprentissages populaires qu'est née la prise révolutionnaire de la place Tahrir.

\section{BIBLIOGRAPHIE}

Abdelfattah, D., 2011. Impacts of Arab Revolts on Migration. CARIM Analytic and Synthetic Notes 2011/68, 17 p. http://www.carim.org/public/workarea/home/Website\%20information/ Literature/asn2011\%20en\%2068.pdf [consulté le 2 juin 2012].

Adonis, 2011. Il n'y a pas eu de révolution arabe. Books, n²6, 29 septembre.

Ambrosetti E., 2011. Égypte, l'exception démographique. Paris, Les Editions de l'INED, 255 p.

Ayari M. B., 2011. Non, les révolutions tunisiennes et égyptiennes ne sont pas des révolutions 2.0. Mouvements, $n^{\circ} 65$, p. 56-61.

Baron B., 2005. Egypt as a woman : nationalism, gender, and politics. Berkeley ; Los Angeles ; London, University of California Press, $287 \mathrm{p}$.

Barthel P.-A., 2011. Une révolution urbaine en marche ? Lectures d'un observateur urbaniste. http:// ebookbrowse.com/pierre-arnaud-barthel-le-caire-en-revolution-doc-d132475657.

Battesti V., Ireton F., 2011. L'Égypte au présent. Inventaire d'une société avant révolution. Arles, Actes Sud, $1179 \mathrm{p}$.

Bayat A., 1996. The coming of a Post-Islamist Society. Critique : Critical MiddleEast Studies, $\mathrm{n}^{\circ} 9$, p. 43-52.

Bayat A., 2010. Life as politics how ordinary people change the Middle East. Stanford, Stanford University Press, 304 p. 
Berry-Chikhaoui I., Deboulet A., Roulleau-Berger L., 2007. Villes internationales : entre tensions et réactions des habitants. Paris, La Découverte, 325 p.

Bertoncello,B., 2010. Diaspora, développement et urbanisation : des Burkinabè de l'étranger, acteurs des projets urbains de Zaca et Ouaga 2000 à Ouagadougou (Burkina Faso). Annales de géographie, $\mathrm{n}^{\circ}$ 675, p. 560-583.

Bozarslan, H., 2011. Sociologie politique du Moyen-Orient. Paris, La Découverte, 125 p.

Burgat F., 2001. De l'islamisme au post islamisme : vie et mort d'un concept. Esprit, n 8-9, p. 82-93.

Clément F., 2011. Le nouveau marché du travail, les conflits sociaux et la pauvreté, in Battesti V., Ireton F., L'Égypte au présent. Inventaire d'une société avant révolution. Arles, Actes Sud, p. 595-624.

Courbage Y., Todd E., 2007. Le rendez-vous des civilisations. Paris, Le Seuil, 184 p.

Denis É., 2011a. Transformation du territoire, urbanisation et libéralisme autoritaire, in Battesti V., Ireton F., L'Égypte au présent. Inventaire d'une société avant révolution. Arles, Actes Sud, p. 75-110.

Denis É., 2011b. La financiarisation du foncier observée à partir des métropoles égyptiennes et indiennes. Tiers Monde, vol. 206, n 2, p. 139-158.

Dorier-Apprill É., 2006. Les échelles du pluralisme religieux en Afrique subsaharienne.

L'Information géographique, vol. 70, $\mathrm{n}^{\circ}$ 4, p. 46-65.

Fargues P., 2006. The demographic benefit of international migration: hypothesis and application to Middle Eastern and North African contexts, in Ozden C., Schiff M, International Migration, Economic Development \& Policy. World Bank, p. 161-182.

Fargues P., 2011. Croissance et mutations démographiques au XX ${ }^{\mathrm{e}}$ siècle, in Battesti V., Ireton F., L'Égypte au présent. Inventaire d'une société avant révolution. Arles, Actes Sud, p. 41-74.

Florin B., 2005. Vivre en parallèle, l'évolution des villes nouvelles du Grand Caire. Annales de la recherche urbaine, $\mathrm{n}^{\circ}$ 98, p. 97-105.

Gauchet M., 1985. Le Désenchantement du monde. Une histoire politique de la religion. Paris, Gallimard, 306 p.

Gruntz, L., Pagès-El Karoui, D., 2013 (à paraître). Migration and family change in Egypt: a comparative approach of social remittances. Migration Letters, Special Issue « Mapping Social Remittances ».

Gonzalez-Quijano Y., Guaaybess T., 2009. Les Arabes parlent aux Arabes : La révolution de l'information dans le monde arabe. Arles, Actes Sud, $272 \mathrm{p}$.

Guaaybess T., 2011. Les médias égyptiens et l'internationalisation des flux, in Battesti V., Ireton F., L'Égypte au présent. Inventaire d'une société avant révolution. Sindbad, Actes Sud, p. 919-952.

Haenni P., 2005. L'islam de marché : l'autre révolution conservatrice. Paris, Seuil, 108 p.

Haenni P., 2006. Islam : Amr Khaled, visionnaire musulman de l'après islamisme. Religioscope. http://religion.info/french/entretiens/article_277.shtml [consulté le 11 janvier 2011].

Haenni, P., Tammam, H., 2011. Islamismes et islamisation : courants et tendances, in Battesti V., Ireton F., L'Égypte au présent. Inventaire d'une société avant révolution. Arles, Actes Sud, p. 887-900.

Herrera, L., 2011. Eduquer la nation : les dilemmes d'un système éducatif à l'ère de la mondialisation, in Battesti V., Ireton F., L'Égypte au présent. Inventaire d'une société avant révolution. Arles, Actes Sud, p. 685-714. 
Hoodfar H., 1997. Between Marriage and the Market: Intimate Politics and Survival in Cairo. Berkeley, University of California Press, $302 \mathrm{p}$.

Ibrahim S., 1993. Les Années de Zeth. Arles, Actes Sud, 345 p.

IOM, 2011. Egypt after January 25: Survey of Youth Migration Intentions. May, 34 p. http://

www.egypt.iom.int/Doc/

IOM\%20\%282011\%29\%20Egypt\%20after\%20January\%2025\%20Survey\%20of\%20Youth\%20Migration\%20Intentions.pdf [consulté le 2 juin 2012].

Kepel, G., 2000. Jihad expansion et déclin de l'islamisme. Paris, Gallimard, 452 p.

Khamis S., 2010. New Media and Social Change in Rural Egypt: Transformations, Paradoxes and Challenges. Arab media and society, 2011. http://www.arabmediasociety.com/countries/ index.php?c_article=238 [consulté le 11 décembre 2011]]

de Koning A., 2006. Café Latte and Caesar Salad : Cosmopolitan Belonging in Cairo's Coffee Shops, in Singerman D., Amar P., Cairo Cosmopolitan. Le Caire, Université Américaine du Caire (AUC), p. 221-234.

Lavergne M., 2011. Monde arabe : des Révolutions en trompe l'œil. Vers un nouveau rendez-vous manqué avec l'Occident? Historiens et géographes, $n^{\circ} 416$, p. 211-220.

Legeay H., 2007. La citoyenneté des bahaïs : l'identité égyptienne en procès. Politique Africaine, $\mathrm{n}^{\circ} 108$, p. 86-105.

Levitt P., 1998. Social remittances: migration driven local-level forms of cultural diffusion. The International Migration Review, vol. 32, n 4, p. 926-948.

Louhichi K., 1997. The Impact of Emigration of the Husband on the Status of the Wife: The Case of Egypt, in Cosio-Zavala F., Women and Families: Evolution of the Status of Women as Factor and Consequence of Changes in Family Dynamics. Paris, CICRED, p. 323-339.

Moriconi-Ebrard F., 2011. Égypte. Tableau de bord de l'urbanisation 1950-2020. Site e-geopolis, http://e-geopolis.eu/menapolis/Tableau_bord_Égypte_final.pdf [consulté le 13 mai 2011].

Müller-Mahn, D., 1998. Du "Zoning familial" au "Zoning économique". La transformation des structures socio-spatiales dans deux villages égyptiens. Revue de Géographie de Lyon, $\mathrm{n}^{\circ} 73$, p. 227-234.

Müller-Mahn D., 2005. Transnational spaces and migrant networks: A case study of Egyptians in Paris. Nord-Süd aktuell, Vol. 19, Issue 2, p. 29-33.

Nehaoua S., 2010. Prédicatrices de salon à Héliopolis : vers la salafisation de la bourgeoisie du Caire ? Le Mouvement Social, vol. 231, n 2, p. 63-76.

Pagès D., Vignal L. 1998. Formes et espaces de la mondialisation en Égypte. Revue Géographique de Lyon, vol. 73, p. 247-258.

Pagès-El Karoui D., 2005. « Le mouled de Sayyid al-Badawî̀ à Tanta. Logiques spatiales et production d'une identité urbaine ", in chiffoleau S., Madoeuf A., Les pèlerinages au Maghreb et au Moyen-Orient. Espaces publics, espaces du public. IFPO, Damas, 2005, pp. 237-264. [En ligne], mis en ligne le 26 août 2010, URL : http://ifpo.revues.org/1208

Pagès-El Karoui D., 2008. Villes du Delta du Nil. Tantâ, Mahalla, Mansûra, cités de la densité. Paris, Karthala, 357 p.

Pagès-El Karoui D., 2012 (à paraître). Les territoires des révolutions arabes. Cybergéo. 
Pagès-El Karoui D, Vignal L., 2011. Les racines de la « révolution du 25 janvier » en Égypte : une réflexion géographique. EchoGéo, URL : http://echogeo.revues.org/12627 [consulté le 11 décembre 2011].

Roussillon A. 1992. Égyptianité, arabité, islamité : la recomposition des référents identitaires. Égypte/Monde arabe, À propos de la nationalité, [En ligne, consulté le 28 juin 2012], URL : http:// ema.revues.org/index316.html

Roy O., 1999a. Pourquoi le post-islamisme ? Revue du monde musulman et de la Méditerranée, vol. 85, $\mathrm{n}^{\circ} 1$, p. 9-10.

Roy O., 1999b. « Le post-islamisme ». Revue du monde musulman et de la Méditerranée, vol. 85, $\mathrm{n}^{\circ}$ 1, p. $11-30$.

Roy O., 2011. Révolution post-islamiste. Le Monde.fr, 12 février, http://abonnes.lemonde.fr/idees/ article/2011/02/12/revolution-post-islamiste_1478858_3232.html [consulté le 3 juillet 2012].

Sanmartin, O., 2011. La "révolution" touristique, in Battesti V., Ireton F., L'Égypte au présent. Inventaire d'une société avant révolution. Sindbad, Actes Sud, p. 581-594.

Tall S. M., 2009. Investir dans la ville africaine : les émigrés et l'habitat à Dakar. Paris, Karthala, 286 p.

Trémoulinas A., 2006. La sociologie des changements sociaux. Paris, La Découverte, 121 p.

Vignal L., 2010. The new territories of tourism in Egypt: a local-global frontier? Cybergeo :

European Journal of Geography, article 509, URL : http://cybergeo.revues.org/23324

Zhuo X., Wellman B., Yu J., 2011. Egypt : The First Internet Revolt ? Peace Magazine, URL :http:// peacemagazine.org/archive/v27n3p06.htm, [consulté le 15 juin 2012].

Zohry A., 2007. Egyptian Irregular Migration to Europe. Migration Letters, vol. 4, n 1, p. 53-63.

\section{NOTES}

1. Mouvement politique, culturel et religieux, initié en Égypte, par des penseurs comme Jamal alDîn al-Afghanî et Muhammad 'Abdû, qui a pour objectif la « renaissance " (nahda) du monde arabe, qui doit se forger une voie originale en se réappropriant la modernité occidentale.

2. D'un point de vue méthodologique, cet article est fondé sur une connaissance profonde de l'Égypte, acquise depuis le milieu des années 1990 pendant une thèse de doctorat sur les villes du delta du Nil, sur des matériaux rassemblés lors de trois enquêtes de terrain (Caire, Delta et Fayoum) en 2008, 2011 et 2012, ainsi que sur des lectures approfondies de travaux académiques variés.

3. Compte tenu de l'importance des déserts, la densité est calculée par rapport au territoire habité, soit environ $35000 \mathrm{~km} 2$.

4. Le terme de réislamisation est ambigu, car il pourrait laisser croire que les Égyptiens ont été désislamisés, ce qui n'est pas le cas. Cependant, l'idéologie nassérienne était fondamentalement laïque, avec l'islam comme un horizon culturel et non comme un référent normatif. Ces processus ne sont pas spécifiques au monde arabe, ils sont notamment très importants en Afrique subsaharienne (Dorier-Aprill, 2006).

5. Callosité qui se forme sur le front, suite au frottement contre le tapis de prière.

6. Tariq Ramadan : «Égypte. Derrière les salafistes, l'Arabie saoudite », Le Point.fr - Publié le 06/12/2011 à 07:30. http://www.lepoint.fr/monde/egypte-tariq-ramadan-derriere-les-salafistesl-arabie-saoudite-06-12-2011-1404244_24.php 
7. Benjamin Barthe, «Les salafistes, adversaires numéro un des Frères musulmans ", Le Monde, 6 décembre 2011.

8. Selon eux, les Frères musulmans en raison de leur engagement politique sont sortis de l'islam.

9. Bien que largement galvaudé, nous utiliserons ici le terme de mondialisation, préféré à celui de néo-libéralisation, dont l'acception, strictement économique, parait trop limité pour rendre compte des phénomènes culturels globalisés. Des chercheurs (Berry-Chikhaoui, Deboulet, Roulleau-Berger, 2007) ont proposé de remplacer mondialisation par internationalisation, mais le concept reste peu employé par la communauté scientifique.

10. Ministère Égyptien des Communications et des Technologies de l'Information, Information and Communication Technology Indicators Bulletin, juin 2011.

11. http://www.socialbakers.com, Consulté le 7 janvier 2012.

12. En juin 2010, à Alexandrie, Khaled Saïd a été tabassé à mort par la police, alors qu'il venait de mettre en ligne une vidéo dénonçant la corruption sévissant dans un commissariat. Les photos de son visage défiguré par les coups qui ont circulé sur Internet puis ont été publiées dans la presse d'opposition ont suscité la colère des Égyptiens. De grandes manifestations ont lieu à Alexandrie et une page Facebook a été créée Nous sommes tous des Khaled Saïd. L'un de ses administrateurs longtemps resté secret n'est autre que Waël Ghoneim, le directeur général de Google Moyen Orient, qui a été l'une des icones de la révolution de 2011.

13. Build, Operate and Transfer

14. En 2006, les villes nouvelles égyptiennes rassemblent près de 850000 personnes (Denis, 2011a).

15. Source : Banque Mondiale. http://data.worldbank.org/indicator/IS.VEH.NVEH.P3

16. Il s'agit de triporteurs importés d'Inde ou auto-rickshaws. Ces mini-taxis qui ne peuvent prendre que deux personnes, plus le chauffeur, circulent notamment dans les petites villes de province et les bourgs périurbains (voir illustration 9).

17. Cette troisième partie repose sur un travail en cours sur les migrations égyptiennes. Débutée en 2011, cette recherche a donné lieu à une quinzaine d'entretiens avec des migrants égyptiens et des acteurs clés (vice-consul de Paris, haut-cadre du Ministre des Affaires étrangères égyptiennes), menés en France et en Égypte.

18. Plusieurs membres de la diaspora ont lancé des initiatives pour soutenir l'économie égyptienne, notamment la Fondation de la place Tahrir, crées par des Egypto-canadiens et des Egypto-américains.

\section{RÉSUMÉS}

Les forces du changement social en Égypte, comme dans le monde arabe, sont restées invisibles pour des nombreux observateurs occidentaux, rendant incompréhensibles et " miraculeux » les soulèvements de 2011. Pour éclairer ces changements en amont de la révolution, nous reviendrons d'abord sur les transformations structurelles, liés à l'individualisation de la société facilitée par l'urbanisation du territoire -, et ses traductions au niveau de la famille et de la religion. Nous mettrons ensuite en relation ces processus avec les mutations économiques globales (néo-libéralisation, règne de la société de consommation et de loisirs, essor des nouvelles technologies). Enfin, nous interrogerons le rôle d'une catégorie d'acteurs, les émigrés, qui apparaissent souvent dans les médias égyptiens comme les boucs-émissaires du changement social. 
In Egypt, as in the Arab world, the strong dynamics of social change have remained invisible for many western observers, for whom the 2011 uprisings were impossible to understand, rather miraculous. This paper aims to explore the changes upstream of the revolution and its new landscapes. It focuses first on the structural changes, i.e. the individualization of the society facilitated by a deep urbanization-, analyzed here at the family and religious levels. It deals also with the role of global economic transformations (liberalization, the rise of consumption and leisure society, new technologies). Then, it questions the role of one category of agents, the migrants, usually depicted in Egyptian media as scapegoats of social change.

\section{INDEX}

Keywords : Egypt, globalization, migration, revolution, social change

Mots-clés : changement social, Égypte, migration, mondialisation, révolution

Thèmes : Sur le Champ - Sur le Terrain

\section{AUTEUR}

\section{DELPHINE PAGÈS-EL KAROUI}

Delphine Pagès-El Karoui, dpages@inalco.fr, est Maître de conférences en géographie à l'INALCO (Institut National des Langues et Civilisations Orientales). Elle a publié récemment :

- Pagès-El Karoui D., 2010. Shisha Cafés in France: Reinventing the Oriental Dream. From

Exoticism to Beurs Integration. Die Erde, $\mathrm{n}^{\circ} 141$ (1-2), Special issue New geographies of migration, p. 31-63.

- Pagès-El Karoui D., 2008. Villes du Delta du Nil. Tantâ, Mahalla, Mansûra, cités de la densité. Paris, Karthala, 357 p.

- Bennafla K., Pagès-El Karoui D., Sanmartin O., 2007. Géopolitique du Maghreb et du Moyen-Orient. Paris, SEDES, $230 \mathrm{p}$. 